\title{
Papeles de Europa
}

ISSN-e 1989-5917

\section{Inversión pública, inversión en defensa y progreso tecnológio: análisis con base en un modelo DSGE}

\author{
José Lorenzo Jiménez ${ }^{1}$, Antonio Fonfría ${ }^{2}$
}

Fecha de recepción: noviembre 2016 Fecha de aceptación: mayo 2017

Resumen. El presente trabajo sostiene la importancia de la inversión en defensa, como parte de la inversión pública, en la potenciación de la base industrial y tecnológica del país y en el desarrollo de sectores productivos innovadores. El análisis se realiza a través de un modelo de equilibrio general dinámico estocástico (DSGE). La principal conclusión que se deduce es que el aumento en la inversión pública, cuando es complementaria del progreso tecnológico asociado a la inversión privada, se traduce en un crecimiento de la producción, el empleo, la inversión y la productividad en el conjunto de la economía. En consecuencia, en la medida que la inversión pública en defensa es generadora de innovación y progreso tecnológico, también contribuye de forma positiva al crecimiento del país.

Palabras clave: Inversión pública; Inversión en defensa; Base Industrial y Tecnológica de la Defensa; Programas de Armamento y Material; Efectos macroeconómicos; Modelos DSGE.

\section{[en] Public investment in defense and technological progress: a DSGE model}

\begin{abstract}
This paper argues the importance of investment in defense, as part of public investment in enhancing the industrial and technological base of the country as well as in the development of innovative productive sectors. The analysis is performed through models of dynamic stochastic general equilibrium (DSGE). The main conclusion is that the increase in public investment, when it is complementary to the technological progress associated with private investment, translates into higher growth of production, employment, investment and productivity in the whole economy. Accordingly, to the extent that public investment in defense is generating innovation and technological progress, also it contributes positively to growth.
\end{abstract}

Key words: Public investment; Defence investment; Defence industrial and technological base; Weapon programs;Macroeconomic effects; DSGE models.

JEL: C68, H56, L64

\section{Introducción}

Considerando la Defensa Nacional como un bien público, la Economía de la Defensa reclama entre sus contenidos, el estudio de aquellas actividades económicas que coadyuvan a dotar a los países de las capacidades necesarias para mitigar los riesgos y amenazas a las que se enfrentan sus ciudadanos, teniendo en cuenta las relaciones existentes entre los presupues- tos disponibles, la tecnología y la industria de la defensa, ecuación de difícil resolución por los numerosos factores, sociales, estratégicos, políticos y económicos que se encuentran implicados.

En la literatura económica, autores como Aschauer (1989) y Barro (1990) han defendido el potencial impacto del gasto público productivo en el crecimiento económico a largo plazo, considerando los determinantes tanto

Ejército del Aire / Ministerio de Defensa

jjimbas@ea.mde.es

2 Universidad Complutense de Madrid

Director de la Cátedra UCM-ISDEFE

afonfria@ccee.ucm.es 
de la inversión pública como de la privada, como generadores de riqueza. Greenwood et al. (1988) se han centrado en el papel que jugaron en el crecimiento económico de EEUU, con posterioridad a Segunda Guerra Mundial, determinadas inversiones realizadas en tecnologías muy específicas. La premisa de la que parten es la de que la introducción de nuevos bienes de capital más eficientes es una fuente importante de variación en la productividad. Este enfoque es un novedoso intento de desagregación o de descomposición de los efectos del tradicional planteamiento del progreso tecnológico neutral propugnado por Hicks. Su modelo se fundamenta en el análisis cuantitativo del cambio tecnológico basado de forma específica en el cambio tecnológico generado por la inversión que recoge con mayor precisión la evolución del crecimiento económico.

Por su parte, Greenwood et al. (1997), mostraron la importancia de la inversión privada que promueve el cambio tecnológico específico asociado a los nuevos bienes de capital. Utilizaron para ello, un modelo de crecimiento de equilibrio general estocástico dinámico (DSGE, en sus siglas en inglés) que incorpora este tipo de cambio tecnológico, y que considera los precios relativos de los bienes de equipo, para identificar el proceso de explicación del crecimiento, provocado por variaciones en la inversión asociadas a tecnologías nuevas y específicas. Muestran, igualmente, que este tipo de cambio tecnológico explica en torno al $30 \%$ de las fluctuaciones del PIB.

En los trabajos citados se desarrolla un modelo DSGE en el que la inversión en cada periodo se transforma en capital en función de la tecnología asociada a él. Es decir, por cada unidad de producción que se destina a la inversión, el capital resultante de la misma depende del cambio tecnológico o carga tecnológica implícita en el mismo. Representan una novedad, en la medida que se introduce el progreso tecnológico especifico a la inversión en la función de acumulación del capital, como un proceso estocástico endógeno asociado a la inversión.

En concreto, el modelo de la teoría del Ciclo Económico Real (RBC en sus siglas en inglés) básico introduce una serie de supuestos muy específicos sobre el proceso de acumulación de capital, entre los que destacan: El ahorro que se transforma directamente en capital a través del proceso de inversión, siendo este proceso irreversible.
La realidad es compleja, y la simplificación anterior ha de reflejar que el progreso tecnológico altera las características del capital a lo largo del tiempo. Es decir, con el transcurso del tiempo, cuando se incorporan nuevos activos de capital a la economía a través del proceso de inversión, estos activos presentan características diferentes a los ya existentes (Hulten (1992), Greenwood et al. (1997), y Torres (2013). En las últimas dos décadas el progreso tecnológico asociado al capital en bienes de equipo viene siendo muy intenso. Hoy día es fácil constatar el cambio radical que están experimentando áreas como las telecomunicaciones o los equipamientos informáticos, los cuales presentan un desarrollo tecnológico muy superior al que muestran otros tipos de capital.

Además, existe un progreso tecnológico adicional, que resulta ser específico a la inversión - Pavitt y Patel (1988)—. De este modo, cuando se incorporan nuevos activos de capital a la economía, estos resultan ser más avanzados que los existentes previamente. Es decir, los activos de capital no son homogéneos con el paso del tiempo, ya que la carga tecnológica implícita en cada uno de ellos es diferente. En este caso, el progreso tecnológico únicamente se traslada a la economía en la medida en que ésta introduce los nuevos activos de capital que ya incorporan dicho progreso tecnológico.

En consecuencia, es importante diferenciar entre progreso tecnológico neutral derivado de cambios en la productividad total de los factores $(\mathrm{PTF})$, frente a progreso tecnológico asociado al proceso de inversión en nuevos activos de capital. Mientras que el primero supone un cambio en el nivel de eficiencia general de la economía, el segundo tipo de progreso tecnológico hace referencia a la cantidad de tecnología que puede ser adquirida con una unidad de producción. Esta tecnología es específica a cada uno de los diferentes activos de capital existentes.

Greenwood, Hercowitz y Huffman (1988) son los primeros en introducir el progreso tecnológico específico a la inversión en la función de acumulación del capital, como un proceso estocástico exógeno asociado a la inversión. . En su trabajo se preguntan por el papel real que juega el cambio tecnológico específico de la inversión, desde el punto de vista cuantitativo, y la principal característica de su modelo es poder mostrar que la producción de bienes de capital se hace cada vez más eficiente con 
el paso del tiempo. Observa que la senda de crecimiento en el equilibrio del modelo tiene la característica de que tanto el stock de equipo existente como la inversión en el nuevo equipo (medido en unidades ajustadas por la calidad) crecen a una tasa más elevada que la producción. Así, se puede ir un paso más adelante, en el sentido de que "es posible obtener inferencias sobre en qué medida el crecimiento del stock del capital se ha debido al cambio tecnológico específico de la inversión versus el crecimiento de la productividad total de los factores, o productividad neutral".

El elemento clave del modelo sería un proceso de acumulación de capital vendría definido como:

$$
K_{t+1}=\left(1-\delta_{K}\right) K_{t}+Z_{t} I_{t}
$$

Donde $\delta_{K}$ es la ratio de depreciación y $Z_{t}$ representa el progreso tecnológico específico a la inversión privada.

Siguiendo a Greenwood et al. (1997), $Z_{t}$ determina la cantidad de capital que puede ser comprada con una unidad de producción, representando, por tanto, el estado actual de la tecnología para producir capital. En el modelo neoclásico estándar se tendría que $=1$ para todo $t$, es decir, la cantidad de capital que puede ser comprada con una unidad de producción final es constante en el tiempo. Sin embargo, en la realidad el precio relativo del capital disminuye en términos generales, lo que evidencia que a lo largo del tiempo podemos comprar una mayor cantidad de capital con la misma cantidad de producción final. Así, cuanto mayor sea Zt mayor es la cantidad de capital que se puede incorporar a la economía con una unidad de inversión, reflejando que la calidad del capital ha aumentado.

Para obtener una medida del progreso tecnológico específico a la inversión, se hace necesario disponer de precios de los activos de capital ajustados por la calidad. Esto es lo que se denomina precios hedónicos, es decir, el precio de un determinado activo de capital cuya calidad se mantiene constante a lo largo del tiempo ${ }^{3}$.

Así, por ejemplo, no podemos comparar el precio de un automóvil hoy respecto al de un automóvil de hace 20 años, dado que la calidad del mismo ha ido variando en el tiempo (el de hace 20 años no tenía ni frenos ABS, ni Airbag, ni dirección asistida, etc.). Para poder realizar dicha comparación tendríamos que disponer de precios corregidos por la calidad del producto y podría resultar el caso de que el automóvil de hoy sea más barato que el de hace 20 años. Este fenómeno se puede observar claramente en el caso de los ordenadores personales.
Por lo anteriormente expuesto, el precio de los activos de capital corregidos por calidad vendría dado por $1 / Z t$. De este modo, el progreso tecnológico implícito vendría determinado por el cociente entre los precios del capital corregidos por calidad y el deflactor del consumo, medido como el índice de precios de los bienes no-duraderos más los servicios, excluyendo los servicios de las viviendas — Torres (2013) - . El hecho de que el desarrollo tecnológico implícito a cada activo de capital sea muy diferente es lo que ha llevado a desagregar el stock de capital, ya que cada uno de ellos lleva pareja una calidad diferente.

En consecuencia, podemos concluir que no es lo mismo hablar de activos de capital en infraestructuras, por ejemplo, en forma de edificios, que evidencian un progreso tecnológico muy lento en el tiempo que de activos de capital en forma de equipos o maquinaria, que presentan una tasa de progreso tecnológico mucho más elevada. Entre los posibles tipos de activos de capital que podemos considerar, que tienen especial relevancia en este último contexto existe una variada gama, que van desde las denominadas tecnologías de la información y el conocimiento (TICs), que incluyen ordenadores, internet, software, equipos de telecomunicaciones, pasando por las tecnologías de carácter aeronáutico espacial, y los modernos y sofisticados sistemas de armas, con guiado laser, satélite, etc. —García Alonso (2010)—. Estos activos de capital muestran unas tasas muy elevadas de progreso tecnológico, por lo que es de esperar que tengan un impacto muy importante sobre el crecimiento de la productividad.

En este sentido, Torres (2013) resalta que existen numerosos estudios que ponen en evidencia el papel fundamental que están jugando las TICs en la recuperación de la productividad desde mediados de los noventa en Estados Unidos y en algunos países de Europa.

Es de señalar que, hasta donde se conoce, no se tiene constancia de la construcción de un modelo DSGE básico, de las características que aquí se formulan. Una aproximación muy importante y novedosa, utilizando modelos DSGE, relacionada con el papel que desempeña el gasto en defensa, sus efectos sobre la seguridad y el ciclo económico, es abordado por F de Córdoba y Torres (2014) para la economía de EE.UU. Dichos autores para analizar el papel que juega el gasto militar y sus particularidades, lo introducen en el 
modelo, pero asociado a la Función de Utilidad de los consumidores. Nuestro análisis, a diferencia del anterior, se centra en analizar la eventual importancia de la inversión en defensa, en su relación con la función de producción agregada de la economía y el posible impacto que se produce como consecuencia de considerar el proceso tecnológico específico asociado a la inversión privada, generado por la inversión militar. Por tanto, nuestra aproximación es diferente, a pesar de utilizar igualmente el análisis de las funciones impulso respuesta (irf) que proporciona la moderna teoría macroeconómica a través de sus modelos DSGE.

El propósito de este trabajo será intentar destacar la importancia de la inversión en defensa, como parte de la inversión pública, en la potenciación de la base industrial y tecnológica del país y en el desarrollo de sectores productivos innovadores, de una forma cuantitativa, empleando el instrumental que hoy ofrece la nueva teoría macroeconómica: los modelos DSGE. Se pretende poner de relieve que no produce los mismos efectos una inversión pública centrada solamente en infraestructuras, que una inversión pública que además contemple una parte específica dedicada a inversión en equipos, sobre todo cuando estos equipos acaparan la mayor parte de progreso tecnológico especifico de la inversión privada. En España, los dos grandes ministerios inversores son el de Fomento (infraestructuras) y el de Defensa (Equipos y grandes sistemas de Armas).

El trabajo se estructura del siguiente modo. En la Sección 2 se plantean diversas cuestiones relacionadas con la inversión pública y el stock de capital tanto público como privado y el estado de la cuestión en los trabajos previos relacionados con nuestro objeto de estudio. En la Sección 3 se contempla un modelo DSGE básico, con unos supuestos muy simplificadores. En la Sección 4, se construye un modelo DSGE, donde se introducen en la función de producción un mayor número de variables. En la Sección 5 se explican y concretan los supuestos de calibración que se utilizan para el anterior modelo, se obtienes las condiciones de equilibrio, y se resuelve y simula el modelo, obteniéndose las funciones de impulso respuesta (irf), correspondientes a las perturbaciones estocásticas introducidas. En la última Sección se resumen los resultados y se destacan algunas conclusiones.

\section{Inversión en defensa, inversión pública y stock de capital físico}

Se considera que el progreso tecnológico específico a la inversión privada juega un papel fundamental que trasciende a la productividad del trabajo y a otras variables de la economía de forma endógena, los cuales tienen efectos sobre la evolución económica. Este progreso tecnológico inducido a través de la inversión tiene efectos diferentes según incida sobre la inversión en infraestructuras (Ipi) o sobre inversiones en bienes de equipo (Ipe).

Además, es necesario poner de relieve que el sector público, a través de inversión pública específica, es oferente de activos de capital que actúan como factores productivos directos y específicos en la economía, (las infraestructuras), y de activos de capital en equipos y sistemas, que tiene importantes efectos indirectos sobre un determinado sector privado ${ }^{4}$. Este stock de capital público en equipos, generado por la inversión en defensa principalmente, contribuye a la formación bruta de capital público. Además, genera un efecto indirecto muy concreto e importante, en la medida que provoca una demanda de bienes, materiales y equipos muy sofisticada, específica y particular, sobre empresas que conforman un tejido industrial de alto contenido tecnológico, y que se mueven en la frontera del conocimiento (la industria aeronáutica y aeroespacial de la defensa, por ejemplo). Y es aquí, donde cobra especial importancia la consideración del progreso tecnológico específico de sus inversiones. Otro efecto adicional que se produce en dicho tejido industrial, y está interrelacionado, es el que también generan tecnologías de uso dual, efectos desbordamiento y elevados spinoff. Estás empresas, además, para sobrevivir o mantenerse en el mercado, tienen que realizar elevadas inversiones en $\mathrm{I}+\mathrm{D}+\mathrm{i}$, lo que las obliga a ser muy competitivas, para tener opciones ante un cliente único muy exigente en materia de defensa: el gobierno.

De forma genérica se puede sostener que, las infraestructuras de redes de carreteras y ferrocarriles, obras hidráulicas, infraestructuras de aeropuertos, etc., se transforman de forma directa en activos de capital público, a través de un proceso de gasto público en inversiones públicas. Este gasto hace posible poner a disposición de los ciudadanos servicios públicos que mejoran

En este trabajo se tratarán ambos tipos de inversiones. 
su función de utilidad, y también de las empresas privadas, aportando posibilidades de desarrollo de una determinada actividad económica, que les reporta unos beneficios extraordinarios intangibles en sus cuentas de resultados, y que probablemente de no existir dichas infraestructuras, no serían viables ni siquiera sus objetivos sociales. (Cassou y Lansing, 1998)

Pero, además, y como efecto indirecto, propicia la actividad empresarial privada necesaria para la provisión de dichas infraestructuras públicas. Este último efecto lleva aparejada, durante el periodo de ejecución de las infraestructuras en cuestión, una determinada generación de medios e inversiones que debe realizar la empresa privada, así como una demanda de fuerza laboral, unida la mayoría de las veces, a nuevos componentes tecnológicos e innovaciones que la empresa privada se tiene que procurar para poder suministrar lo requerido por el gobierno.

El otro gran componente de la inversión pública es la inversión en activos físicos de capital proporcionados por bienes de equipo. Por lo que respecta a los relativos a la defensa, según la SEC 2010 todos los gastos públicos relacionados con las adquisiciones de grandes sistemas de $\operatorname{armas}^{5}$, así como los gastos en I+D asociados a los mismos, son inversiones públicas que contribuyen a la formación bruta de capital público. Así, es necesario plantearse de nuevo la cuestión,

El efecto directo e inmediato de esa inversión pública para el caso de bienes de equipo asociados con la adquisición de grandes sistemas de armas es dotar al país de un determinado grado de seguridad y defensa, que genera un nivel de disuasión ante las eventuales amenazas que todo país tiene que hacer frente. Del nivel de disuasión obtenido dependerá una parte, no solo de la seguridad nacional, sino también de la estabilidad y de las expectativas para generar confianza en las eventuales inversiones y de las alianzas de todo tipo que se puedan mantener con los países del entorno, con los que normalmente se realizan intercambios económicos, reduciendo así la incertidumbre y la inestabilidad. Estos factores son clave para propiciar la creación de un tejido productivo atractivo que propicie el crecimiento económico ${ }^{6}$.

Por ejemplo aviones de combate o transporte, Fragatas, carros blindados, misiles, etc.

Este aspecto ya se reflejaba por Adam Smith al decir que la seguridad es de mayor importancia que la opulencia.
La medición de esa contribución se realiza de una forma similar a la señalada para las inversiones públicas en infraestructuras, pero con unas peculiaridades que las hacen diferentes por los potenciales efectos multiplicadores indirectos que generan. Como antes ya se ha destacado, la provisión por parte de las empresas privadas de equipos asociados a las adquisiciones de grandes sistemas de armas, a diferencia de la provisión de infraestructuras, requiere de unas capacidades y tecnologías que se encuentran en los límites de la frontera del conocimiento. La supervivencia de esas empresas, para ser competitivas radica en realizar y disponer de inversiones que le permitan estar en un mercado muy complejo por lo especifico y sofisticado de las tecnologías. Es decir, son demandantes de un permanente progreso tecnológico específico en sus inversiones privadas, que les pueda garantizar su supervivencia, el cual, la mayoría de las veces genera uso dual —en el ámbito civil también-, efectos de desbordamiento y learning by doing.

Con carácter general, la medición de la aportación de la inversión pública al proceso productivo consiste en considerar el sector público como oferente de un factor productivo a través de la inversión pública en activos de capital - Torres (2013) - Estos activos de capital, en los que se emplea la inversión pública, son de dos tipos: los asociados a la inversión pública y los que constituyen la inversión en bienes de equipo - formación bruta de capital en sistemas de armas, equipos asociados y la $\mathrm{I}+\mathrm{D}+\mathrm{i}$ unida a ellos- - . La forma de introducir al gobierno en la economía cerrada que se pretende modelizar afecta a la productividad de los factores y distorsiona sus precios. La economía como veremos en el apartado siguiente está constituida por tres tipos de agentes: los consumidores, las empresas y el gobierno. La función de producción en dicha economía contiene tres factores productivos: trabajo, capital privado y capital público.

Ya desde los 70 hay trabajos que incorporan el capital público - básicamente en infraestructuras - , a la función de producción - Arrow y Kurz (1970) o Pestieau (1974) Con el estudio de Barro (1990), se produce un aumento del interés por introducir en los modelos de crecimiento el capital público como input en la función de producción con rendimientos constantes a escala. En este sentido, el análisis de Mera (1973) para la economía japonesa, calcula funciones de producción del 
tipo Cobb-Douglas en las que incluye el capital público, obteniendo un valor medio de la elasticidad del nivel de producción respecto al capital público de 0,2 .

El trabajo de Torres (2013) considera que Finn (1993) y Cassou y Lansing (1998) constituyen excepciones por cuanto analizan la importancia del capital público usando modelos de equilibrio general. Por este motivo, en este trabajo se procede a realizar un análisis basado en un modelo DSGE que introduce el sector público en orden a permitir cuantificar la importancia de la inversión pública sobre el crecimiento económico de la economía.

Es de resaltar, que hay importantes diferencias entre los valores de la elasticidad del nivel de producción respecto al capital público, cuando esta se obtiene con base en modelos de equilibrio general (DSGE) respecto de aquellos otros modelos en los que las estimaciones se realizan únicamente considerando la función de producción agregada de forma individual. En este sentido, Hulten y Schawb (1993), destacan que la función de producción agregada forma parte de un sistema más amplio, en el cual tanto la variable output como los variables inputs se determinan de forma endógena. Esto supone que los resultados obtenidos de la estimación de funciones de producción individuales puedan estar potencialmente sujetos a problemas de sesgo, dado que deberían ser estimados dentro de un sistema ecuaciones, en el marco de un modelo de optimización dinámica de equilibrio en una economía en su conjunto, con todos los agentes que interactúan entre sí. En este caso todas las variables de la función de producción son endógenas y se determinan de forma simultánea"

2. Modelo DSGE con perturbación de la inversión pública pero no contempla progreso tecnológico especifico en la inversión privada (Modelo T)

Aunque en fechas relativamente recientes ha surgido una cada vez más extensa literatura sobre el tema, aún no existe un consenso sobre la importancia cuantitativa del stock de capital público con relación al nivel de producción de una economía. No obstante, en el caso de España, estudios recientes, basados en modelos DSGE, particularmente en el denominado modelo REMS (Boscá, et al., 2011, Capitulo 5, pag. 142), se aprecian evidencias de que la inversión pública puede generar un efecto positivo sobre el nivel de actividad.

El modelo de equilibrio general dinámico estocástico (DSGE) que aquí se analiza y resuelve es el planteado por Torres (2013), Capítulo 9, pag.187-203. Dicho modelo incorpora la existencia de capital público, con el objetivo de analizar la importancia del mismo sobre el crecimiento económico, demás variables económicas que intervienen en la economía que modeliza.

El desarrollo de este modelo se realiza a fin de compararlo con el que posteriormente se pasará a desarrollar (Modelo J). A efectos de la exposición que sigue, pasamos a denominarlo Modelo T, y utilizando el código Dynare aportado por dicho autor, se procede a su resolución y análisis. Se basa fundamentalmente en la inspección del comportamiento de las funciones irf, relativas a la perturbación de la productividad total de los factores, que contempla función de producción agregada, y la perturbación que se introduce para analizar los efectos de una variación sobre la inversión pública en capital. De dicho modelo se destacan algunas de sus particularidades:

La función de producción que relaciona el nivel de producción agregado de la economía está integrada por tres factores de producción: trabajo, capital privado y capital público:

$$
Y_{t}=A_{t} K_{t}^{\alpha_{1}} G_{t}^{\alpha_{2}} L_{t}^{\alpha_{3}}
$$

Para la producción del bien privado final, $\mathrm{Y}$, se requiere los servicios del trabajo, L, y de los dos tipos de capital considerado: capital privado, K, y capital público, G. $A_{t}$ es una medida de la productividad total de los factores, y representa una perturbación estocástica de la productividad total de los factores, y viene dada por:

$$
\ln A_{t}=\left(1-\rho_{A}\right) \ln \bar{A}+\rho_{A} \ln A_{t-1}+\varepsilon_{t}^{A}
$$

$\alpha_{1}, \alpha_{2} y \alpha_{3}$ son los parámetros tecnológicos asociados a cada uno de los factores productivos. Se considera la existencia de rendimientos constantes respecto a los factores productivos, por lo que, en equilibrio, si no existiesen inputs públicos, los beneficios de las empresas serían nulos.

El problema al que se enfrentan las familias o consumidores consiste en maximizar el valor de su función de utilidad: 


$$
\operatorname{Maximizar}_{\left\{C_{t}, L_{t}\right\}_{t=0}^{\infty}} \sum_{t=0}^{\infty} \beta^{t}\left[\gamma \log C_{t}+(1-\gamma) \log \left(1-L_{t}\right)\right]
$$

sujeta a la restricción presupuestaria del consumidor representativo que viene dada por:

$$
\left(1-\tau_{t}^{e}\right) C_{t}+I_{t}=\left(1-\tau_{t}^{l}\right) W_{t}^{e} L_{t}+\left(1-\tau_{t}^{k}\right) R_{t}^{e} K_{t}+T_{t}
$$

La restricción presupuestaria indica que el consumo más la inversión no pueden exceder la suma de las rentas provenientes del trabajo y de capital, netas de impuestos, más las transferencias. Los precios relativos de los factores privados resultantes del modelo, dados por $W_{t}^{e} \mathrm{y} R_{t}^{e}$, van a ser superiores a los correspondientes a sus productividades marginales respectivas, y que viene representadas por $W_{t} y R_{t}$.

El stock de capital privado se mueve de acuerdo con la siguiente ley de formación:

$$
K_{t+1}=\left(1-\delta_{K}\right) K_{t}+I_{t}
$$

Donde $\delta_{K}$ es la tasa de depreciación del capital privado y donde $I_{t}$ es la inversión privada bruta.

Las empresas resuelven el problema de encontrar los valores óptimos para la utilización de los factores productivos privados, capital y trabajo, dada la presencia de los factores productivos públicos. La empresa representativa utiliza la función de producción del tipo CobbDouglas, anteriormente planteada en (1). Se considera que $\alpha_{1}+\alpha_{2}+\alpha_{3}=1$, con lo que las empresas obtienen un beneficio positivo, igual a la diferencia entre el valor de su producción y el coste de los factores productivos privados.

El gobierno fija unos impuestos sobre el consumo de bienes y servicios, sobre los ingresos del capital y sobre los ingresos del trabajo, con el objeto de financiar una secuencia exógena de transferencias y financiar una secuencia de inversión pública. Es decir, los impuestos se utilizan tanto para realizar transferencias no distorsionantes a los agentes, como para financiar la inversión pública en activos de capital fijo en infraestructuras. El gobierno, por tanto, obtiene recursos de la economía a través de la fijación de impuestos sobre el consumo, y sobre los ingresos del trabajo y del capital, siendo los tipos marginales efectivos, $\tau_{t}^{c}, \tau_{t}^{l}, \tau_{t}^{k}$, respectivamente. La restricción presupuestaria del gobierno en cada periodo vendría dada por lo tanto por,

$$
\tau_{t}^{c} C_{t}+\tau_{t}^{l} W_{t}^{e} L_{t}+\tau_{t}^{k}\left(R_{t}^{e}-\delta_{K}\right) K_{t}=T_{t}+I_{G, t}
$$

donde $I_{G, t}$ es la inversión pública.

Por lo tanto, el gobierno, por un lado, obtiene unos ingresos a partir de impuestos $\mathrm{y}$, por otro, destina dichos ingresos a gastos en capital público y a transferencias. Utiliza los ingresos que obtiene a través de los impuestos, para financiar la inversión en capital público, que aumenta la productividad total de los factores, entrando en la función de producción como un factor productivo adicional a los factores privados, junto con la realización de transferencias a las familias. Se supone que la restricción presupuestaria del gobierno se cumple periodo a periodo, a través de la devolución de los in- gresos provenientes de los impuestos no gastados en inversión pública a las familias, a través de transferencias, $T_{t}$.

El capital público que se genera se usa en el proceso productivo por parte de las empresas, como un factor productivo adicional a los privados. El hecho de que el factor productivo público se use sin coste por parte de las empresas provoca que estas obtengan beneficios extraordinarios, que se reparten como retribución adicional de los factores de producción privados, lo que significa que el precio pagado por los inputs privados va a ser superior a su productividad marginal. La inversión pública 
va destinada a la acumulación de capital público, en un proceso similar al del capital privado,

$$
I_{G, t}=G_{t}-\left(1-\delta_{G}\right) G_{t-1}
$$

Por tanto, el capital público viene dado por:

$$
G_{t}=\left(1-\delta_{G}\right) G_{t-1}+I_{G, t}
$$

donde $\delta_{G}$ es la tasa de depreciación del capital público.

Un elemento clave del modelo planteado por Torres (2013) se encuentra en determinar cómo es la decisión de inversión en capital público. Se supone que la inversión pública es una proporción aleatoria del nivel de producción de la economía, tal que:

$$
I_{G, t}=B_{t} \theta_{G} Y_{t}
$$

donde $\theta_{G}>0$, es una determinada proporción del nivel de producción, y $B_{t}$ representa una perturbación estocástica asociada al proceso de inversión en capital público. Siendo:

$$
\ln B_{t}=\left(1-\rho_{B}\right) \ln \bar{B}+\rho_{B} \ln B_{t-1}+\varepsilon_{t}^{B}
$$

Donde, $\rho_{B}$, es el parámetro autorregresivo asociado a la perturbación estocástica, cuyo valor absoluto es $\left|\rho_{B}\right|<1$, con objeto de asegurar la estacionariedad del proceso; $\bar{B}$ es el valor estacionario, o de equilibrio de la perturbación
$B_{t} ; \varepsilon_{t}^{B}$ es una función de distribución de probabilidad normal $\left(\varepsilon_{t}^{B} \sim N\left(0, \sigma_{B}^{2}\right)\right.$, siendo $\sigma_{B}^{2} \mathrm{su}$ varianza.

Una vez concretados los comportamientos optimizadores de los agentes que intervienen en su modelo, se define y plantea el equilibrio del modelo, se resuelve el problema de optimización dinámica del consumidor mediante la obtención de las condiciones de primer orden, se resuelve el problema de maximización al que tiene que hacer frente la empresa, y se formulan las ecuaciones que resuelven el modelo. Los parámetros considerados vienen dados por:

$$
\left\{\alpha_{1}, \alpha_{2}, \alpha_{3}, \alpha, \delta_{K}, \delta_{G}, \beta, \gamma, \theta_{G}, \tau^{c}, \tau^{l}, \tau^{k}\right\}
$$

A dichos parámetros se les asignan unos determinados valores por el procedimiento de calibración.

En el trabajo de Torres (2009), se calibra un modelo de equilibrio general con capital público para la economía española, y obtiene un valor de 0,068 para la elasticidad de la producción respecto al capital público, un valor que, según afirma el autor, es similar al obtenido por De la Fuente (2003), por Mas et al (1996) $y$ por otros trabajos similares que utilizan un enfoque basado en modelos de equilibrio general.

Para el Modelo T que se está considerando, Torres (2013) supone, por razones de simplificación, que el parámetro tecnológico asociado al capital público en la función de producción es de 0,1 . A modo de resumen, en la siguiente Tabla 1 se muestran los valores calibrados.

Tabla 1. Parámetros calibrados (Torres, 2013)

\begin{tabular}{|c|l|c|}
\hline Parámetro & \multicolumn{1}{|c|}{ Definición } & Valor \\
\hline$\alpha_{1}$ & Parámetro tecnológico capital privado & 0,315 \\
\hline$\alpha_{2}$ & Parámetro tecnológico empleo & 0,585 \\
\hline$\alpha_{3}$ & Parámetro tecnológico capital público & 0,100 \\
\hline$\alpha$ & Proporción rentas del capital & 0,350 \\
\hline$\beta$ & Factor de descuento & 0,970 \\
\hline$\gamma$ & Parámetro preferencias & 0,450 \\
\hline$\delta_{K}$ & Tasa de depreciación capital privado & 0,060 \\
\hline$\delta_{G}$ & Tasa de depreciación capital público & 0,020 \\
\hline$\theta_{G}$ & Proporción de Inversión Pública & 0,050 \\
\hline$\rho_{A}$ & Parámetro autorregresivo PTF & 0,950 \\
\hline$\sigma_{A}$ & Desviación estándar PTF & 0,001 \\
\hline$\tau^{C}$ & Impuesto sobre el consumo & 0,116 \\
\hline$\tau^{l}$ & Impuesto sobre las rentas del trabajo & 0,348 \\
\hline$\tau^{k}$ & Impuesto sobre las rentas del capital & 0,225 \\
\hline
\end{tabular}




\subsection{Efectos de una perturbación en la productividad total de los factores (PTF)}

Es de resaltar que, en este tipo de modelos, dado su nivel de complejidad, no suele haber una solución analítica inmediata, dado que, en la mayoría de los casos, simplemente no existe. Por ello, lo habitual es proceder a resolverlo empleando métodos numéricos ${ }^{7} \mathrm{y}$ a obtener una simulación del mismo. Además, y como es sabido, el análisis macroeconómico consiste, en gran medida, en producir o simular una determinada perturbación, en un momento del tiempo concreto, y analizar cuáles son sus efectos sobre las distintas variables endógenas a lo largo de un periodo determinado.

En la actualidad, la forma más habitual de representar los efectos de una perturbación es a través de las denominadas funciones de impulso respuesta (irf). Estas suponen una representación gráfica con relación al tiempo de las desviaciones de cada variable respecto de su estado estacionario inicial, una vez que se produce una determinada perturbación.

Como explica Torres (2013), una vez resuelto el modelo se pueden realizar una gran variedad de experimentos y aplicaciones empíricas que nos permiten explicar la dinámica de las diferentes variables macroeconómicas de la economía simulada. El ejercicio consiste en estudiar cómo responden las variables del modelo ante una o varias perturbaciones, calculando las desviaciones de las distintas variables consideradas respecto a su valor en estado estacionario. Además, se está interesado en estudiar como el sistema vuelve a su estado estacionario inicial, o bien en cómo se mueve hacia un nuevo estado estacionario, en función de si la perturbación tiene efectos transitorios o permanentes.

Como señalan Kidland y Prescot (1982) y Long y Plosser (1983) en el análisis de la literatura de los modelos de ciclo económico real (RBC), los ciclos están generados por perturbaciones exógenas a la función de producción. El mecanismo implícito en estos modelos es el siguiente:

La perturbación que recibe la función de producción hace que los agentes alteren sus decisiones de consumo e inversión, debido a su comportamiento optimizador en un entorno dinámico.

$>\quad$ La perturbación que recibe la función de producción también altera la productividad marginal del trabajo, lo que afecta a las decisiones trabajo-ocio.

$>$ La acumulación de capital introduce un elemento de persistencia, incluso en el caso en que la perturbación que afecta al nivel de producción esté no correlacionada serialmente.

En consecuencia, se puede obtener un conjunto de efectos que, en términos generales, son similares a los observados durante los ciclos económicos. Este ejercicio es ilustrativo del funcionamiento del modelo y de las diferentes relaciones que existen entre las distintas variables.

En el Modelo $T$ al que nos venimos refiriendo, y una vez resuelto, se procede a la obtención de las funciones impulso respuesta derivadas tanto de la perturbación asociada a la productividad total de los factores, $A_{t}$, como de la perturbación producida como consecuencia de una variación en la inversión pública por parte del gobierno, $B_{t}$. En el Modelo T, los efectos de una perturbación asociada a la productividad total de los factores sobre las variables que intervienen en la economía modelizada se muestran en la Figural mediante los gráficos que reflejan las funciones impulso respuesta para cada una de las variables del modelo.

La Figura 1 muestra los efectos de la perturbación de la PTF a lo largo de los 40 periodos posteriores a que se produzca la perturbación, se supone que también que inicialmente se produce un aumento de la PTF del 1\%, dada la persistencia que se le ha supuesto para el proceso que sigue la PTF $\left(A_{t}\right)$, se supone que disminuye de valor de forma paulatina en el tiempo, ya que como se ha indicado se ha modelizado siguiendo un proceso de Markow, o proceso autorregresivo de orden uno, con un parámetro relativamente elevado, cercano a la unidad $(0,95)$ indicando con ello un elevado grado de autocorrelación. 
Figura 1. Perturbación asociada al Productividad Agregada Total (PTF).
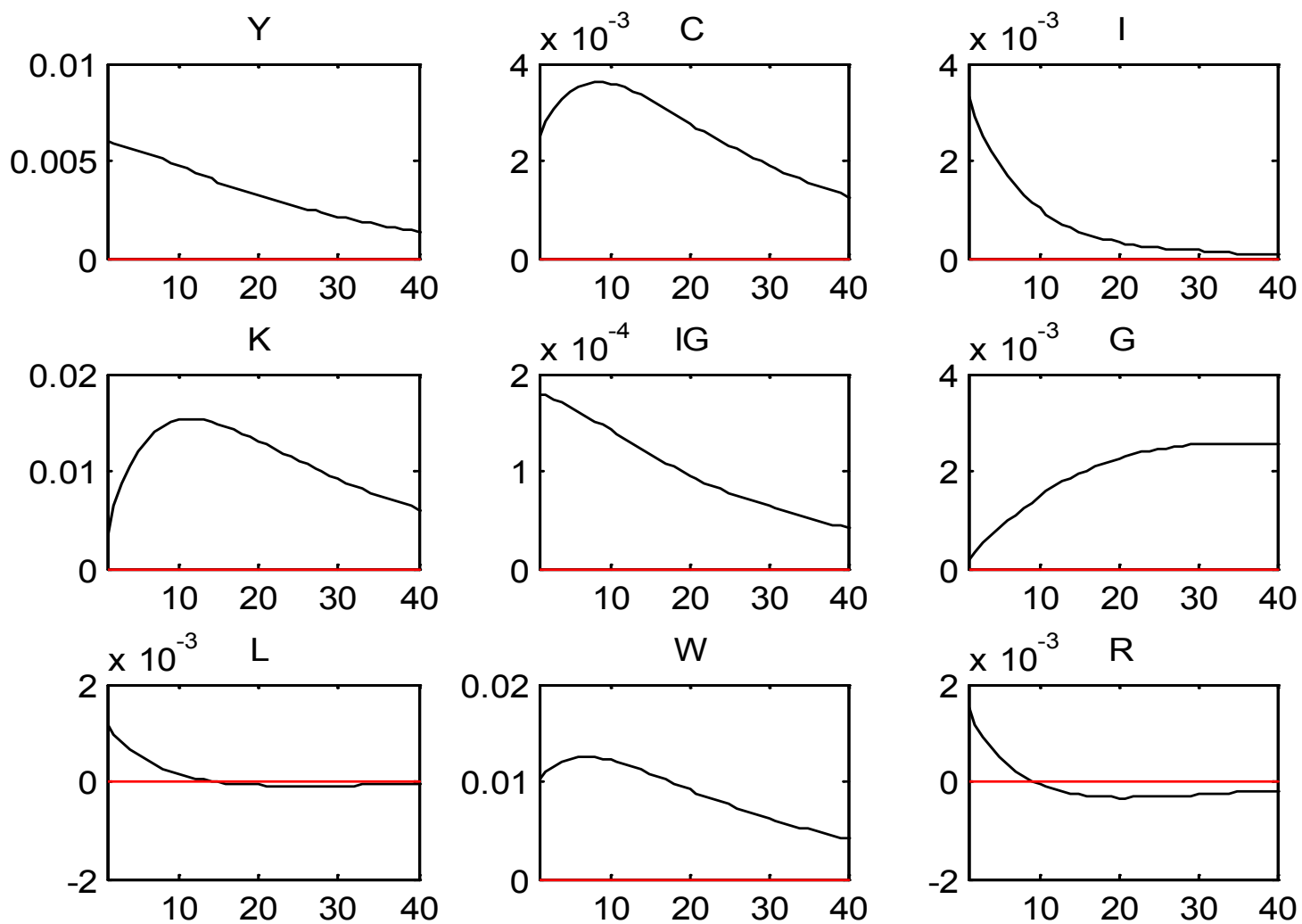

Mediante un análisis detenido de los gráficos de la Figura1, se observa la evolución de las respuestas que experimentan las variables ante la citada perturbación $A_{t}$, Las particularidades más destacables son las siguientes:

1. Para el nivel de producción (Y) se observa que se produce un incremento instantáneo, mostrando una desviación de forma positiva respecto a su valor de estado estacionario. Posteriormente, la desviación positiva comienza a disminuir, pero mostrando una importante persistencia en el tiempo. De hecho, después de haber transcurrido 20 periodos, el nivel de producción sigue siendo un $0.5 \%$ superior al valor de estado estacionario. Por tanto, como era de esperar una perturbación de productividad positiva provoca un efecto positivo sobre el nivel de producción. Es importante resaltar que el nivel de producción aumenta inicialmente, sin que varíe la cantidad de capital y con un leve aumento en el factor productivo trabajo. Esto se debe a que la perturba- ción supone un aumento instantáneo de la PTF, que se traslada directamente a la producción.

2. El consumo también aumenta de forma instantánea respecto a su valor de estado estacionario, pero en menor proporción. Posteriormente, dicha desviación sigue aumentando hasta alcanzar un nivel máximo pasados 10 periodos, para ir disminuyendo posteriormente. Observamos así que la respuesta del consumo ante esta perturbación tiene forma de campana. Este comportamiento del consumo viene explicado tanto por el comportamiento del nivel de producción como por el comportamiento del nivel de inversión. La inversión aumenta de forma instantánea como consecuencia del choque de productividad, para posteriormente ir disminuyendo rápidamente hacia su valor de equilibrio.

3. El stock de capital (K) de la economía también muestra una función impulso respuesta en forma de campana. Así, inicialmente el aumento de la inversión provoca también un aumento de stock 
de capital (la inversión neta es positiva). Sin embargo, a medida que la inversión disminuye, el stock de capital alcanza un máximo a partir del cual comienza a descender, pero siempre por encima de su valor de estado estacionario.

4. El efecto sobre el empleo (L) es muy limitado. De hecho, el empleo aumenta inicialmente, aunque en un porcentaje muy reducido (aproximadamente un $0,2 \%$ ) para posteriormente disminuir, incluso a valores ligeramente inferiores a su estado estacionario.

5. En relación con el precio de los factores de producción, observamos lo siguiente:

i) El salario (W) aumenta tras la perturbación, hasta alcanzar un máximo, a partir del cual disminuye, pero siempre por encima de su valor de estado esta- cionario, reflejando el efecto positivo de la productividad agregada sobre la productividad marginal del trabajo.

ii) El tipo de interés real (R) experimenta una ligera variación positiva inicialmente, dado el aumento de la productividad marginal del capital. No obstante lo anterior, con posterioridad podemos observar que disminuye ligeramente por debajo de su valor de estado estacionario como consecuencia del proceso de acumulación del capital generado.

Así mismo, los efectos que produce en el modelo $\mathrm{T}$, ante una perturbación en la inversión pública, se muestra en los gráficos que reflejan las funciones impulso respuesta para cada una de las variables del modelo, como se aprecia en la Figura 2:

Figura 2. Perturbación sobre la inversión pública en capital público Modelo T.
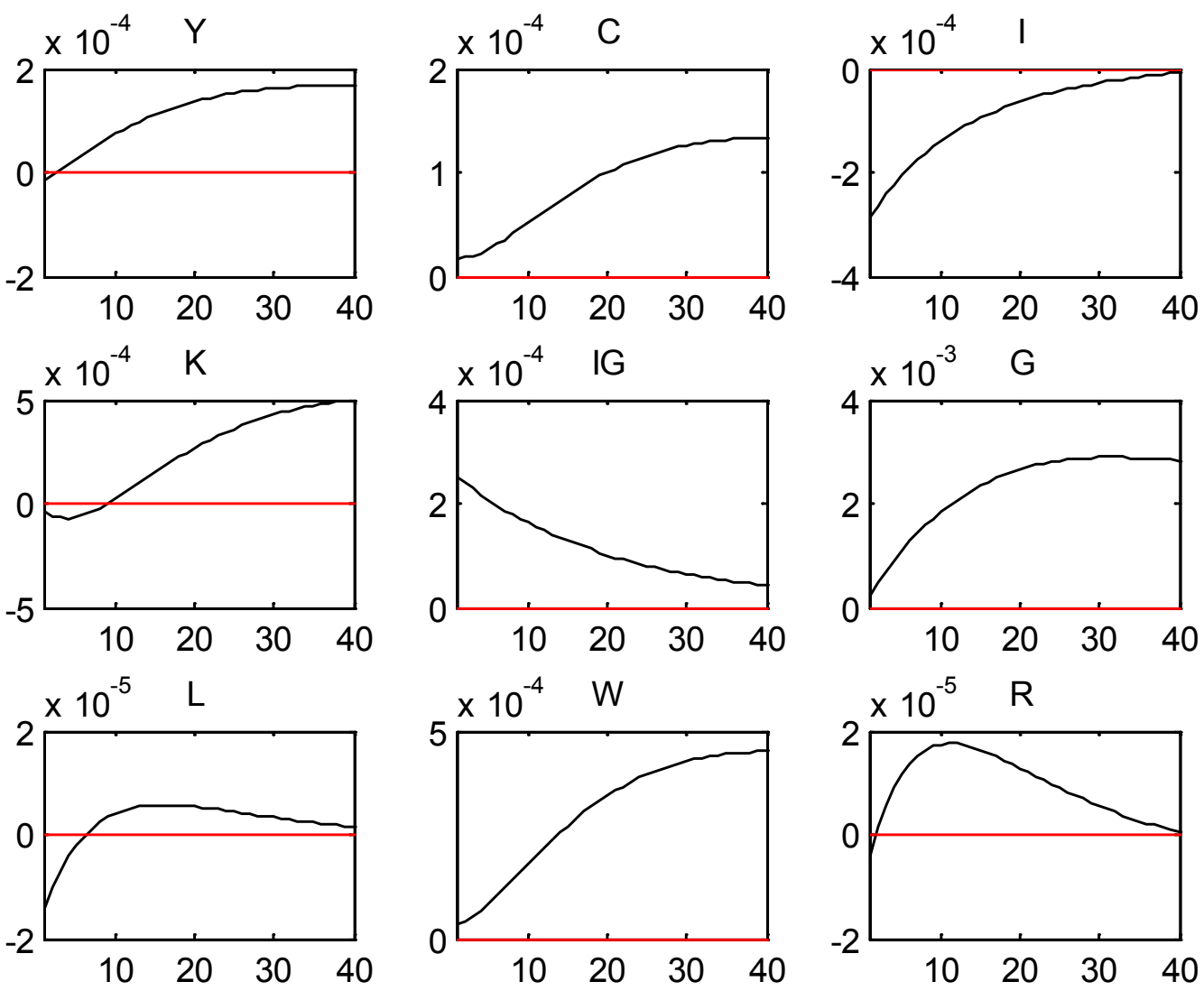

Es evidente, que a la vista de los gráficos de la Figuras 1 y 2 los efectos que producen las perturbaciones y difieren notablemente. Como señala Torres (2013), y en base al mode- lo DSGE que estamos considerando, se puede observar en la Figura 2 que la perturbación estocástica sobre la inversión pública $\left(B_{t}\right)$, provoca, para cada una de las siete variables endó- 
genas, dadas por: $Y_{t}, C_{t}, I_{P, t}, K_{P, t}, L_{t}, R_{t}, W_{t}$ y las variables exógenas, dadas por: $I_{G, t}, K_{G, t}$, las siguientes particularidades:

1. Un aumento de la inversión pública, como resultado de la perturbación de en un $1 \%$, da lugar a una mayor acumulación del capital público. Se supone que el proceso estocástico asociado a la inversión pública muestra una elevada persistencia en el tiempo, similar al proceso que ha seguido la PTF.

2. Se observa que la inversión pública que aumenta inicialmente para ir disminuyendo de forma gradual, hasta alcanzar su valor de estado estacionario, transcurridos más de 40 periodos, y que como se puede ver en la Tabla 3, dicho valor es un 4,9\% de la producción. Este efecto induce un proceso de acumulación de capital público, que alcanza valores superiores a su estado estacionario, hasta que comienza a disminuir por el efecto de la depreciación.

3 . El consumo privado aumenta inicialmente, como consecuencia de dicha perturbación de la inversión pública.

4. Este mecanismo instantáneo inicial provocado por la inversión pública, supone que al mismo tiempo se produzca una disminución de la inversión privada. Esta disminución de la inversión privada provoca una simultánea disminución inicial del stock de capital privado, que a su vez va a dar lugar a una ligera disminución también inicial del nivel de producción.
5. Con posterioridad, la inversión privada trascurridos unos periodos vuelve a aumentar, coincidiendo con que también aumenta el consumo privado, dando lugar a un proceso de acumulación de capital privado.

6. El aumento del capital público junto con el aumento del capital privado es lo que explica el aumento del nivel de producción sostenido, transcurrido el impacto inicial.

7. A nivel del empleo, se observa que la perturbación de la inversión pública provoca una disminución inicial, pero que posteriormente se produce un aumento, aunque con efectos cuantitativos muy limitados.

8. Se observa que a nivel salarios, se produce un aumento debido a que la mayor dotación de capital público aumenta las rentas que reciben el factor productivo mano de obra.

9. De igual modo, a nivel tipo de interés se produce un aumento, debido a que la mayor dotación de capital público aumenta las rentas que recibe el factor productivo del capital.

Otros resultados destacables obtenidos tras la resolución del Modelo T, además de las referidas funciones irf, son las siguientes funciones de transición y de política, para cada una de las respectivas variables (Tabla 2). La Tabla 3 muestra los valores del estado estacionario o estado de equilibrio de la economía que se pretende modelizar, dada la calibración de los parámetros del modelo.

Tabla 2. Funciones de Decisión y Funciones de transición

\begin{tabular}{|c|c|c|c|c|c|c|c|c|c|c|c|}
\hline \multicolumn{12}{|c|}{ POLICY AND TRANSITION FUNCTIONS } \\
\hline & $\mathbf{Y}$ & C & 1 & $\mathbf{K}$ & IG & G & $\mathbf{L}$ & w & $\mathbf{R}$ & A & B \\
\hline Constant & 0.560215 & 0.442422 & 0.089782 & 1.962 .454 & 0.028011 & 1.400 .478 & 0.243878 & 1.493 .113 & 0.099911 & 1.000 .000 & 1.000 .000 \\
\hline (correction) & 0.000024 & -0.000024 & 0.000047 & 0.000049 & 0.000001 & 0.000001 & 0.000018 & -0.000046 & 0.000004 & 0 & 0 \\
\hline $\mathrm{K}(-1)$ & 0.053370 & 0.107413 & -0.056711 & 0.885958 & 0.002669 & 0.002669 & -0.027198 & 0.308789 & -0.041394 & 0 & 0 \\
\hline$G(-1)$ & 0.049284 & 0.022347 & 0.024473 & 0.026937 & 0.002464 & 0.982464 & 0.006909 & 0.089062 & 0.008790 & 0 & 0 \\
\hline$A(-1)$ & 0.688522 & 0.264645 & 0.389450 & 0.423876 & 0.034426 & 0.034426 & 0.116341 & 1.122 .873 & 0.122800 & 0.950000 & 0 \\
\hline$B(-1)$ & -0.002188 & 0.002178 & -0.030866 & -0.004366 & 0.026500 & 0.026500 & -0.001628 & 0.004137 & -0.000390 & 0 & 0.950000 \\
\hline e & 0.724760 & 0.278574 & 0.409948 & 0.446186 & 0.036238 & 0.036238 & 0.122464 & 1.181 .972 & 0.129263 & 1.000 .000 & 0 \\
\hline $\mathbf{u}$ & -0.002303 & 0.002293 & -0.032490 & -0.004596 & 0.027894 & 0.027894 & -0.001714 & 0.004355 & -0.000411 & 0 & 1.000 .000 \\
\hline thetag & -0.008785 & 0.008748 & -0.577285 & -0.017533 & 0.559751 & 0.559751 & -0.006537 & 0.016612 & -0.001567 & 0 & 0 \\
\hline tauc & -0.358891 & -0.039101 & -0.301846 & -0.319790 & -0.017945 & -0.017945 & -0.267063 & 0.678623 & -0.064009 & 0 & 0 \\
\hline taul & -0.610552 & -0.066519 & -0.513505 & -0.544032 & -0.030528 & -0.030528 & -0.454332 & 1.154 .483 & -0.108893 & 0 & 0 \\
\hline tauk & -0.013828 & 0.013769 & -0.026906 & -0.027598 & -0.000691 & -0.000691 & -0.010290 & 0.026148 & -0.002466 & 0 & 0 \\
\hline
\end{tabular}


Tabla 3. Valores en el estado estacionario

\begin{tabular}{|c|c|c|}
\hline \multicolumn{3}{|c|}{ RESULTADOS EN EL ESTADO ESTACIONARIO } \\
\hline VARIABLE & VALOR & Ratio respecto a Y \\
\hline Y & 0,560191 & 1 \\
\hline C & 0,442446 & 0,78981276 \\
\hline I & 0,0897348 & 0,160186079 \\
\hline K & 1,96241 & \\
\hline IG & 0,0280095 & 0,049999911 \\
\hline G & 1,40048 & \\
\hline L & 0,24386 & \\
\hline W & 1,49316 & \\
\hline R & 0,0999069 & \\
\hline A & 1 & \\
\hline B & 1 & \\
\hline
\end{tabular}

En síntesis, Torres (2013), tras plantear y explicar su modelo, destaca que, en última instancia, la introducción de capital público tiene una influencia muy limitada sobre el comportamiento a lo largo del ciclo de las principales variables macroeconómicas, pero importantes implicaciones sobre el comportamiento de la economía en el largo plazo. La inversión pública aumenta la cantidad de factores productivos disponibles en la economía. En este contexto, señala que los ejercicios más relevantes consistirían en cuantificar cuál es la aportación del capital público al crecimiento económico y determinar cuál es el stock de capital púbico óptimo en una economía.

3. Modelo DSGE con capital publico y privado, en infraestructuras y equipos, y con perturbación de la inversión pública que contempla el progreso tecnológico especifico de la inversión privada (Modelo J)

En este apartado se aborda el estudio de otro modelo DSGE, que pasamos a denominar Modelo $\mathrm{J}$, en el que se contempla una función de producción agregada que incluye stock de capital privado y stock de capital público. Ambos tipos de capital se desagregan en infraestructuras y en equipos. A estos últimos se les atribuye un proceso tecnológico específico derivado de las nuevas inversiones. En particular, al stock de capital público en equipos se le asocia con las inversiones públicas en equipos y sistemas de armas, la cual se considera influye en una proporción determinada de la inversión privada en equipos, con las características propias del progreso tecnológico específicas. Con base en la resolución de dicho modelo, se trata de dar respuesta a las diferencias existentes con el modelo $\mathrm{T}$ con respecto a las irf obtenidas, en relación a las irf que se calculan con un nuevo Modelo J, resultado de introducir perturbaciones asociadas a la productividad total de los factores, perturbaciones asociadas a la inversión en progreso tecnológico especifico de la inversión privada, y perturbaciones en la inversión pública.

Recordamos a modo de introducción a este modelo, y siguiendo a Torres (2013), que la ecuación clásica del proceso de acumulación de capital supone que periodo a periodo cada unidad que se gasta en inversión se transforma en capital y que el capital es una variable que se mantiene homogénea a través del tiempo. Es decir, periodo a periodo, es una parte de la producción la que se transforma en capital. Sin embargo, en la práctica, el progreso tecnológico altera las características del capital a lo largo del tiempo. Cuando incorporamos nuevos activos de capital a la economía a través del proceso de inversión, estos activos presentan características diferentes a los ya existentes, es decir, no son homogéneos con el transcurso del tiempo.

Sin embargo, en la realidad existe un progreso tecnológico adicional, que resulta ser específico a la inversión. De este modo, cuando se incorporan nuevos activos de capital a la economía, estos resultan ser más avanzados que los existentes previamente. Es decir, los activos de capital no son homogéneos con el paso del tiempo, ya que la carga tecnológica implícita en cada uno de ellos es diferente. En 
este caso, el progreso tecnológico únicamente se traslada a la economía en la medida en que ésta introduce los nuevos activos de capital que ya incorporan dicho progreso tecnológico.

Greewood et al (1997) plantea un modelo DSGE donde el capital privado se descompone en Infraestructuras, y en Equipos. Introducen el papel de gobierno, solo a efectos de recaudación de impuestos distorsionantes, que posteriormente trasfiere en su totalidad a las familias. Además, y esto es lo más relevante, introduce los mecanismos mediante los cuales la inversión privada genera un progreso tecnológico específico.

El modelo J que se construye y analiza en este apartado es una modificación del modelo desarrollado por Greenwood, Hercowitz y Huffman (1988) y Greenwood, Hercowitz y Krusell (1997). En él, además de las perturbaciones asociadas a la productividad total de los factores, , y la correspondiente a una perturbación en la inversión pública, que afecta tanto a infraestructuras como a equipos, se introduce otra perturbación adicional, , la cual está asociada con el progreso tecnológico especifico generado por la inversión privada en bienes de equipos. Ahora la inversión en equipos, tanto pública como privada, en cada momento del tiempo se transforma en capital en función de la tecnología asociada a dicho capital. En otras palabras, por cada unidad de producción que se destina a la inversión, el capital resultante de la misma depende de la carga tecnológica implícita en el mismo. Además, se introduce el potencial efecto de la inversión pública en bienes de equipo y sistemas de armas de alta tecnología, como una proporción relacionada con dicha perturbación generadora de progreso tecnológico especifico, en la medida que se supone que también los bienes de equipo que proporciona la industria del sector defensa son resultado y consecuencia de la demanda de un cliente único denominado Ministerio de Defensa.

Bajo el marco simplificador que se le supone al modelo, se pasa a estudiar un modelo de equilibrio general dinámico estocástico, que pretende ser más realista que el modelo $\mathrm{T}$ analizado previamente. Como se ha dicho, el modelo contará con tres agentes: los consumidores o familias, las empresas y el gobierno. En él se introduce el progreso tecnológico asociado a la inversión privada, al tiempo que se plantea un escenario adicional en el que la inversión pública en equipos se llevará a efecto teniendo en cuenta dicha perturbación tecnológica Z. El modelo contempla mercados competitivos, y tendrá, además del factor trabajo, cuatro factores productivos de capital. Dos para el sector privado, representado respectivamente, por el stock de capital generado por las inversiones privadas en infraestructuras, y las inversiones privadas en equipos. Y dos para el sector público, que representan respectivamente, las infraestructuras públicas y el stock de capital público en equipos y sistemas. Como ya se dijo antes, el análisis de esta aproximación se realiza para el caso de España, y se consideran, respectivamente, al Ministerio de Fomento (infraestructuras) y al de Defensa (equipos y grandes sistemas de armas), como los dos ministerios generadores de los citados stocks de capital público. La función de producción agregada, para este Modelo J, será de la forma:

$$
Y_{t}=A_{t}\left[K_{e, t}^{\alpha_{1 e}} K_{s, t}^{\alpha_{1 s}}\right]\left[G_{e, t}^{\alpha_{G e}} G_{s, t}^{\alpha_{G s}}\right] L_{t}^{\alpha_{L}}
$$

\subsection{Los consumidores, el gobierno y las empresas}

Los consumidores tienen la misma función de Utilidad definida para el modelo T. Su restricción presupuestaria, no obstante, será la que más abajo se indica. Las empresas tratan de maximizar beneficios sometidos a esta la función de producción, cuyos factores y tecnología son los mostrados en (5). El papel del gobierno será en que también mostramos seguidamente.
Tanto consumidores como empresas representativas, intentaran optimizar sus respectivas funciones objetivo, sometidas a sus correspondientes restricciones presupuestarias. Se obtendrán las condiciones de equilibrio del modelo y se procederá a su calibración, con una tabla de parámetros y criterios que también se indicarán más abajo. Posteriormente, se calculará las condiciones iniciales de estabilidad o condiciones de equilibrio, y con Matlab/Dynare se procederá a su resolución y simulación. 
Como se ha citado, en el modelo se introducen tres perturbaciones estocásticas, una asociada a la PTF, dada por, $A_{t}$, otra asociada al progreso tecnológico específico de la inversión privada, $Z_{t}$, y otra asociada a la posibilidad de adquisiciones del gobierno de inversión pública, en las dos modalidades antes indicadas, y que más abajo se concretarán. Las funciones irf, que se obtienen son muy interesantes, en la medida que ponen de relieve las consecuencias del progreso tecnológico específico de la inversión privada, y la importancia de su consideración, con independencia de los muchos supuestos que se van a tener que asumir, dada la escasez de datos estadísticos disponibles. Aun así, es un ejercicio que permite plantearse nuevas vías de investigación, en orden a conocer con más precisión el verdadero impacto que tiene la inversión en equipos de defensa, y su relación con el progreso tecnológico especifico de la inversión privada.

Se considera que el sector público es oferente de un factor productivo, constituido por determinados activos de capital, a través de la inversión pública. Es decir, se introduce al gobierno, a través del gasto en la inversión pública en activos de capital, (infraestructuras y equipos y grandes sistemas) lo cual va a tener efectos sobre la productividad de los factores privados.

La función de producción de la economía, como se ha dicho, contendrá cinco factores productivos: trabajo, $\mathrm{L}_{\mathrm{t}}$, capital privado en infraestructuras, $\mathrm{K}_{\mathrm{s}, \mathrm{t}}$, y capital privado en equipos, $\mathrm{K}_{\mathrm{s}, \mathrm{t}}, \mathrm{y}$ capital público en infraestructuras, $\mathrm{G}_{\mathrm{s}, \mathrm{t}}$, y capital público en equipos y grandes sistemas operativos, $G_{e, t}$. Se considera que el gobierno fija unos impuestos sobre el consumo de bienes y servicios, sobre los ingresos del capital (equipos e infraestructuras) y sobre los ingresos del trabajo, con el objeto de financiar una secuencia exógena de transferencias, $\left\{\mathrm{T}_{\mathrm{t}}\right\}_{\mathrm{t}=0}^{\infty} \mathrm{y}$ financiar una secuencia de inversión pública, $\left\{\mathrm{I}_{\mathrm{g}, \mathrm{t}}\right\}_{\mathrm{t}=0}^{\infty}$.
El modelo, como se adelantó para el modelo $\mathrm{T}$, introduce una característica particular en términos de cómo se distribuyen los beneficios extraordinarios que obtienen las empresas como consecuencia del capital público. El gobierno se comporta como un agente doble, por un lado, obtiene unos ingresos a partir de impuestos y, por otro, destina dichos ingresos a gastos en capital público y a transferencias. El gobierno usa los ingresos que obtiene a través de los impuestos, además de emplearlos para la realización de transferencias a las familias, para financiar la inversión en capital público. Esta inversión pública, que aumenta la productividad total de los factores, entra en la función de producción como un factor productivo adicional a los factores privados. Así, podemos ver que la función de producción agregada de la economía toma la forma, señalada en (5):

$$
Y_{t}=A_{t}\left[K_{e, t}^{\alpha_{1 e}} K_{s, t}^{\alpha_{1 s}}\right]\left[G_{e, t}^{\alpha_{G e}} G_{s, t}^{\alpha_{G s}}\right] L_{t}^{\alpha_{L}}
$$

La restricción presupuestaria del gobierno se cumple, periodo a periodo, mediante la devolución a las familias de los ingresos provenientes de los impuestos distorsionadores no gastados en inversión pública, a través de transferencias, $\mathrm{T}_{\mathrm{t}}$. Este supuesto se realiza con objeto de simplificar el análisis. Por otra parte, como señala Torres (2013), los resultados derivados del modelo no cambian significativamente si se introduce la posibilidad de desequilibrios fiscales periodo a periodo. El gobierno obtiene recursos de la economía a través de la fijación de impuestos sobre el consumo, y sobre los ingresos del trabajo y sobre los ingresos provenientes del capital, siendo los tipos marginales efectivos aplicados, $\tau_{\mathrm{t}}^{\mathrm{c}}$, $\tau_{\mathrm{t}}^{\mathrm{l}}, \tau_{\mathrm{t}}^{\mathrm{k}}$, respectivamente. Por todo ello, la restricción presupuestaria del gobierno en cada periodo vendría dada por,

$$
\tau_{\mathrm{t}}^{\mathrm{c}} \mathrm{C}_{\mathrm{t}}+\tau_{\mathrm{t}}^{\mathrm{l}} \mathrm{W}_{\mathrm{t}}^{\mathrm{e}} \mathrm{L}_{\mathrm{t}}+\tau_{\mathrm{t}}^{\mathrm{k}}\left[\left(\mathrm{R}_{\mathrm{et}}^{\mathrm{e}}-\delta_{\mathrm{Ke}}\right) \mathrm{K}_{\mathrm{et}}+\left(\mathrm{R}_{\mathrm{st}}^{\mathrm{e}}-\delta_{\mathrm{Ks}}\right) \mathrm{K}_{\mathrm{st}}\right]=I_{G e, t}+I_{G s, t}+\mathrm{T}_{\mathrm{t}}
$$

donde $I_{G e, t}+I_{G s, t}$ es la inversión pública. Planteado de otra manera, la restricción del gobierno ha de verificar:

$$
\mathrm{I}_{\mathrm{Ge}, \mathrm{t}}+\mathrm{I}_{\mathrm{Gs}, \mathrm{t}}+\mathrm{T}_{\mathrm{t}}=\tau_{\mathrm{t}}^{\mathrm{c}} \mathrm{C}_{\mathrm{t}}+\tau_{\mathrm{t}}^{\mathrm{l}} \mathrm{W}_{\mathrm{t}}^{\mathrm{e}} \mathrm{L}_{\mathrm{t}}+\tau_{\mathrm{t}}^{\mathrm{k}}\left[\left(\mathrm{R}_{\mathrm{et}}^{\mathrm{e}}-\delta_{\mathrm{Ke}}\right) \mathrm{K}_{\mathrm{et}}+\left(\mathrm{R}_{\mathrm{st}}^{\mathrm{e}}-\delta_{\mathrm{Ks}}\right) \mathrm{K}_{\mathrm{st}}\right]
$$

La inversión pública va destinada a la acumulación de capital público en equipos y en sistemas, y a la acumulación de capital público en infraes- tructuras. Es un proceso similar al de la formación de capital privado en equipos, y en infraestructuras. Así, La inversión pública vendrá dada por: 
(7) $\mathrm{Z}_{\mathrm{t}} \mathrm{I}_{\mathrm{Ge}, \mathrm{t}}=\mathrm{G}_{\mathrm{et}}-\left(1-\delta_{\mathrm{Ge}}\right) \mathrm{G}_{\mathrm{e}, \mathrm{t}-1}$

Siendo $\mathrm{Z}_{\mathrm{t}}$ una proporción del factor que representa el progreso tecnológico asociado a la inversión privada. Este supuesto se hace en base a considerar que la inversión pública que realiza el gobierno en equipos y sistemas operativos las realiza a un núcleo de empresas que constituyen un tejido industrial muy específico y que sus características básicas son que el gobierno es el cliente único y para ellas este cliente es básicamente el mayor receptor de su oferta ${ }^{8}$. Es decir, existe una relación especial por la cual las inversiones en alta tecnología que debe hacer la empresa van dirigidas a este proveedor único, el gobierno. Por tanto, el capital público o la formación del capital público que se forma es consecuencia de un gasto público específico destinado a la inversión en equipos de alta sofisticación y elevada tecnología, y vienen dado por la siguiente ley de formación:

$$
\mathrm{G}_{\mathrm{et}}=\left(1-\delta_{\mathrm{Ge}}\right) \mathrm{G}_{\mathrm{et}-1}+\mathrm{Z}_{\mathrm{t}} \mathrm{I}_{\mathrm{Ge}, \mathrm{t}}
$$

donde $\delta_{\mathrm{Ge}}$ es la tasa de depreciación del capital público en equipos, como se ha dicho. Siendo $\mathrm{Z}_{\mathrm{t}}$ una proporción del factor que representa el progreso tecnológico asociado a la inversión privada.

$$
\ln Z_{t}=\left(1-\rho_{Z}\right) \ln \bar{Z}+\rho_{\mathrm{Z}} \ln Z_{t-1}+\varepsilon_{t}^{Z}
$$

El elemento clave del modelo está en determinar cómo es la decisión de inversión en capital público. Procedemos a diferenciar tres supuestos concretos de aplicación de la inversión pública:

* Inversión pública en infraestructuras, considerada como una proporción aleatoria del nivel de producción, representada por $B_{t}$.

$$
\begin{gathered}
\mathrm{I}_{\mathrm{Gs}, \mathrm{t}}=\mathrm{B}_{\mathrm{t}} \theta_{\mathrm{G}} \mathrm{Y}_{\mathrm{t}} \\
\mathrm{G}_{\mathrm{st}}=\left(1-\delta_{\mathrm{Gs}}\right) \mathrm{G}_{\mathrm{st}-1}+\mathrm{I}_{\mathrm{Gs}, \mathrm{t}} \\
\mathrm{I}_{\mathrm{Gs}, \mathrm{t}}=\mathrm{G}_{\mathrm{st}}-\left(1-\delta_{\mathrm{Gs}}\right) \mathrm{G}_{\mathrm{s}, \mathrm{t}-1} \\
\ln \mathrm{B}_{\mathrm{t}}=\left(1-\rho_{\mathrm{B}}\right) \ln \overline{\mathrm{B}}+\rho_{\mathrm{B}} \ln \mathrm{B}_{\mathrm{t}-1}+\varepsilon_{\mathrm{t}}^{\mathrm{B}}
\end{gathered}
$$

* Inversión pública en equipos y grandes sistemas de armas, considerada como una proporción aleatoria del progreso tecnológico específico de la inversión privada, representada por $\mathrm{Z}_{\mathrm{t}}$.

$$
\begin{gathered}
\mathrm{I}_{\mathrm{Ge}, \mathrm{t}}=\mathrm{Z}_{\mathrm{t}} \theta_{\mathrm{G}} \mathrm{Y}_{\mathrm{t}} \\
\mathrm{G}_{\mathrm{et}}=\left(1-\delta_{\mathrm{Ge}}\right) \mathrm{G}_{\mathrm{et}-1}+\mathrm{Z}_{\mathrm{t}} \mathrm{I}_{\mathrm{Ge}, \mathrm{t}} \\
\mathrm{Z}_{\mathrm{t}} \mathrm{I}_{\mathrm{Ge}, \mathrm{t}}=\mathrm{G}_{\mathrm{et}}-\left(1-\delta_{\mathrm{Ge}}\right) \mathrm{G}_{\mathrm{e}, \mathrm{t}-1} \\
\ln \mathrm{Z}_{\mathrm{t}}=\left(1-\rho_{\mathrm{Z}}\right) \ln \overline{\mathrm{Z}}+\rho_{\mathrm{Z}} \ln \mathrm{Z}_{\mathrm{t}-1}+\varepsilon_{\mathrm{t}}^{\mathrm{Z}}
\end{gathered}
$$

* Inversión pública considerada de forma simultánea, tanto como inversión en equipos como inversión en infraestructuras.

La inversión pública en equipos se considera que es una proporción aleatoria del progreso tecnológico específico de la inversión privada, $\mathrm{Z}_{\mathrm{t}}$. Es decir, se considera el supuesto aplicable al caso de aquellas inversiones públicas asociadas con la obtención de producto con un elevado componente tecnológico y de mucha $\mathrm{I}+\mathrm{D}+\mathrm{i}$. Este equipo se adquiere bajo la exigencia a un determinado tejido industrial que trabaja en la frontera del conocimiento, y que denominaremos inversión pública en Equipos y grandes sistemas operativos. La inversión pública en infraestructuras sería el otro tipo de inversión pública considerado, y se caracteriza porque tiene un bajo o casi nulo nivel de progreso tecnológico especifico demandado a las empresas constructoras.

La redistribución de las rentas públicas entre los factores privados es la segunda posibilidad a la que anteriormente hemos hecho referencia en el modelo $\mathrm{T}$, y es la estrategia que se sigue en nuestro análisis. Consiste en que las rentas generadas por el capital público pueden distribuirse entre los factores privados, en función de la participación de éstos sobre las rentas totales.

La idea es que las rentas que genera el capital público, y que no aparecen como tales, se encuentran incorporadas en las rentas de los factores productivos privados. Así, del problema de maximización de la empresa, obtenemos las siguientes condiciones de primer orden (FOC):

$$
\begin{aligned}
& \text { (12) } \mathrm{W}_{\mathrm{t}}=\alpha_{\mathrm{L}} A_{\mathrm{t}} \mathrm{K}_{\mathrm{e}, \mathrm{t}}^{\alpha_{\mathrm{e}}} \mathrm{K}_{\mathrm{s}, \mathrm{t}}^{\alpha_{\mathrm{s}}} \mathrm{G}_{\mathrm{e}, \mathrm{t}}^{\alpha_{\mathrm{Ge}}} \mathrm{G}_{\mathrm{s}, \mathrm{t}}^{\alpha_{\mathrm{Gs}}} \mathrm{L}_{\mathrm{t}}^{\alpha_{\mathrm{L}}-1} \\
& \mathrm{R}_{\mathrm{et}}=\alpha_{\mathrm{e}} A_{\mathrm{t}} \mathrm{K}_{\mathrm{e}, \mathrm{t}}^{\alpha_{\mathrm{e}}-1} \mathrm{~K}_{\mathrm{s}, \mathrm{t}}^{\alpha_{\mathrm{s}}} \mathrm{G}_{\mathrm{e}, \mathrm{t}}^{\alpha_{\mathrm{Ge}}} \mathrm{G}_{\mathrm{s}, \mathrm{t}}^{\alpha_{\mathrm{Gs}}} \mathrm{L}_{\mathrm{t}}^{\alpha_{\mathrm{L}}} \\
& \mathrm{R}_{\mathrm{st}}=\alpha_{\mathrm{s}} \mathrm{A}_{\mathrm{t}} \mathrm{K}_{\mathrm{e}, \mathrm{t}}^{\alpha_{\mathrm{e}}} \mathrm{K}_{\mathrm{s}, \mathrm{t}}^{\alpha_{\mathrm{s}}-1} \mathrm{G}_{\mathrm{e}, \mathrm{t}}^{\alpha_{\mathrm{Ge}}} \mathrm{G}_{\mathrm{s}, \mathrm{t}}^{\alpha_{\mathrm{Gs}}} \mathrm{L}_{\mathrm{t}}^{\alpha_{\mathrm{L}}}
\end{aligned}
$$

De las cuales se deduce que: 


$$
\begin{aligned}
& \mathrm{W}_{\mathrm{t}}=\alpha_{\mathrm{L}} A_{\mathrm{t}} \mathrm{K}_{\mathrm{e}, \mathrm{t}}^{\alpha_{\mathrm{e}}} \mathrm{K}_{\mathrm{s}, \mathrm{t}}^{\alpha_{\mathrm{s}}} \mathrm{G}_{\mathrm{e}, \mathrm{t}}^{\alpha_{\mathrm{Ge}}} \mathrm{G}_{\mathrm{s}, \mathrm{t}}^{\alpha_{\mathrm{Gs}}} \mathrm{L}_{\mathrm{t}}^{\alpha_{\mathrm{L}}-1}=\alpha_{\mathrm{L}} \frac{\mathrm{Y}_{\mathrm{t}}}{\mathrm{L}_{\mathrm{t}}} \\
& \mathrm{R}_{\mathrm{et}}=\alpha_{\mathrm{e}} \mathrm{A}_{\mathrm{t}} \mathrm{K}_{\mathrm{e}, \mathrm{t}}^{\alpha_{\mathrm{e}}-1} \mathrm{~K}_{\mathrm{s}, \mathrm{t}}^{\alpha_{\mathrm{s}}} \mathrm{G}_{\mathrm{e}, \mathrm{t}}^{\alpha_{\mathrm{Ge}}} \mathrm{G}_{\mathrm{s}, \mathrm{t}}^{\alpha_{\mathrm{Gs}}} \mathrm{L}_{\mathrm{t}}^{\alpha_{\mathrm{L}}}=\alpha_{\mathrm{e}} \frac{\mathrm{Y}_{\mathrm{t}}}{\mathrm{K}_{\mathrm{et}}} \\
& \mathrm{R}_{\mathrm{st}}=\alpha_{\mathrm{s}} \mathrm{A}_{\mathrm{t}} \mathrm{K}_{\mathrm{e}, \mathrm{t}}^{\alpha_{\mathrm{e}}} \mathrm{K}_{\mathrm{s}, \mathrm{t}}^{\alpha_{\mathrm{s}}-1} \mathrm{G}_{\mathrm{e}, \mathrm{t}}^{\alpha_{\mathrm{Ge}}} \mathrm{G}_{\mathrm{s}, \mathrm{t}}^{\alpha_{\mathrm{Gs}}} \mathrm{L}_{\mathrm{t}}^{\alpha_{\mathrm{L}}}=\alpha_{\mathrm{s}} \mathrm{K}_{\mathrm{tt}}
\end{aligned}
$$

Por otra parte, calculando la derivada de la función de producción con respecto al capital público obtenemos:

$$
\begin{gathered}
\mathrm{G}_{\mathrm{t}}^{\alpha_{\mathrm{G}}}=\mathrm{G}_{\mathrm{e}, \mathrm{t}}^{\alpha_{\mathrm{Ge}}} \mathrm{G}_{\mathrm{s}, \mathrm{t}}^{\alpha_{\mathrm{Gs}}} \alpha_{\mathrm{Gs}} \\
\alpha_{\mathrm{G}}=\alpha_{\mathrm{Ge}}+\alpha_{\mathrm{Gs}}
\end{gathered}
$$

$$
\frac{\partial Y_{t}}{\partial G_{e t}}=\alpha_{G e} A_{t} K_{e, t}^{\alpha_{e}} K_{s, t}^{\alpha_{s}} G_{e, t}^{\alpha_{G e}-1} G_{s, t}^{\alpha_{G s}} L_{t}^{\alpha_{3}}
$$

(14-bis)

$$
\frac{\partial Y_{t}}{\partial G_{s t}}=\alpha_{G s} A_{t} K_{e, t}^{\alpha_{e}} K_{s, t}^{\alpha_{s}} G_{e, t}^{\alpha_{G e}} G_{s t}^{\alpha_{G S}-1} L_{t}^{\alpha_{3}}
$$

Es necesario destacar que la expresión (14) y (14-bis) no son una condición del modelo, dado que no hay agentes que reclamen las rentas generadas por el capital público, tanto en equipos como en infraestructuras. Bajo nuestro supuesto tampoco $\mathrm{W}_{\mathrm{t}}, \mathrm{R}_{\mathrm{et}}, \mathrm{y} \mathrm{R}_{\mathrm{st}}$ serían los precios relativos de los factores productivos privados de equilibrio, dado que en ese caso una parte de las rentas privadas correspondería al capital público. Esto es debido a que las empresas no pagan por el uso del capital público, lo que equivaldría a un menor coste de los factores privados que el correspondiente a su productividad marginal. Así, del problema de maximización de beneficios, obtendríamos que las rentas de cada factor productivo serían:

$$
\begin{gathered}
\mathrm{W}_{\mathrm{t}} \mathrm{L}_{\mathrm{t}}=\alpha_{\mathrm{L}} \mathrm{Y}_{\mathrm{t}} \\
\mathrm{R}_{\mathrm{et}} \mathrm{K}_{\mathrm{et}}=\alpha_{\mathrm{e}} \mathrm{Y}_{\mathrm{t}} \\
\mathrm{R}_{\mathrm{st}} \mathrm{K}_{\mathrm{st}}=\alpha_{\mathrm{s}} \mathrm{Y}_{\mathrm{t}} \\
\frac{\partial \mathrm{Y}_{\mathrm{t}}}{\partial \mathrm{G}_{\mathrm{et}}} \mathrm{G}_{\mathrm{et}}=\alpha_{\mathrm{Ge}} \mathrm{A}_{\mathrm{t}} \mathrm{K}_{\mathrm{e}, \mathrm{t}}^{\alpha_{\mathrm{e}}} \mathrm{K}_{\mathrm{s}, \mathrm{t}}^{\alpha_{\mathrm{s}}} \mathrm{G}_{\mathrm{e}, \mathrm{t}}^{\alpha_{\mathrm{Ge}}} \mathrm{G}_{\mathrm{s}, \mathrm{t}}^{\alpha_{\mathrm{Gs}}} \mathrm{L}_{\mathrm{t}}^{\alpha_{\mathrm{L}}}=\alpha_{\mathrm{Ge}} \mathrm{Y}_{\mathrm{t}} \\
\frac{\partial \mathrm{Y}_{\mathrm{t}}}{\partial \mathrm{G}_{\mathrm{st}}} \mathrm{G}_{\mathrm{st}}=\alpha_{\mathrm{Gs}} \mathrm{A}_{\mathrm{t}} \mathrm{K}_{\mathrm{e}, \mathrm{t}}^{\alpha_{\mathrm{e}}} \mathrm{K}_{\mathrm{s}, \mathrm{t}}^{\alpha_{\mathrm{s}}} \mathrm{G}_{\mathrm{e}, \mathrm{t}}^{\alpha_{\mathrm{Ge}}} \mathrm{G}_{\mathrm{st}}^{\alpha_{\mathrm{Gs}}} \mathrm{L}_{\mathrm{t}}^{\alpha_{\mathrm{L}}}=\alpha_{\mathrm{Gs}} \mathrm{Y}_{\mathrm{t}}
\end{gathered}
$$

Dado que para las empresas el coste por el uso del capital público es cero, la suma de las anteriores expresiones sería inferior al nivel de producción de la economía. De hecho, las empresas generarían beneficios extraordinarios en la magnitud;

$$
\begin{gathered}
\frac{\partial Y_{t}}{\partial G_{t}} G_{t}=\alpha_{G} A_{t} K_{e, t}^{\alpha_{e}} K_{s, t}^{\alpha_{s}} G_{t}^{\alpha_{G}} L_{t}^{\alpha_{3}} \\
G_{t}^{\alpha_{G}}=G_{e, t}^{\alpha_{G e}} G_{s, t}^{\alpha_{G s}} \\
\alpha_{G}=\alpha_{G e}+\alpha_{G s}
\end{gathered}
$$

dado que estas rentas del gobierno no son imputadas a los propietarios de este factor, ya que habitualmente el gobierno no carga el precio que cubre los costes de los servicios que oferta a partir de la contribución del capital público. En nuestro caso, suponemos que las rentas generadas por los inputs públicos se distribuyen entre las rentas de los inputs privados, de forma que las rentas generadas por los factores privados de equilibrio, capital (Equipos e infraestructuras) y mano de obra, vendrían dadas por: 


$$
\begin{aligned}
\mathrm{R}_{\mathrm{Et}}^{\mathrm{e}} \mathrm{K}_{\mathrm{Et}} & =\alpha_{\mathrm{E}} \mathrm{A}_{\mathrm{t}} \mathrm{K}_{\mathrm{e}, \mathrm{t}}^{\alpha_{\mathrm{e}}} \mathrm{K}_{\mathrm{s}, \mathrm{t}}^{\alpha_{\mathrm{s}}} \mathrm{G}_{\mathrm{t}}^{\alpha_{\mathrm{G}}} \mathrm{L}_{\mathrm{t}}^{\alpha_{\mathrm{L}}}+\mathrm{s}_{\mathrm{e}} \alpha_{\mathrm{Ge}} \mathrm{A}_{\mathrm{t}} \mathrm{K}_{\mathrm{e}, \mathrm{t}}^{\alpha_{\mathrm{e}}} \mathrm{K}_{\mathrm{s}, \mathrm{t}}^{\alpha_{\mathrm{s}}} \mathrm{G}_{\mathrm{t}}^{\alpha_{\mathrm{Ge}}} \mathrm{G}_{\mathrm{s}, \mathrm{t}}^{\alpha_{\mathrm{Gs}}} \mathrm{L}_{\mathrm{t}}^{\alpha_{3}} \\
\mathrm{R}_{\mathrm{St}}^{\mathrm{e}} \mathrm{K}_{\mathrm{st}} & =\alpha_{\mathrm{S}} \mathrm{A}_{\mathrm{t}} \mathrm{K}_{\mathrm{e}, \mathrm{t}}^{\alpha_{\mathrm{e}}} \mathrm{K}_{\mathrm{s}, \mathrm{t}}^{\alpha_{\mathrm{s}}} \mathrm{G}_{\mathrm{t}}^{\alpha_{\mathrm{G}}} \mathrm{L}_{\mathrm{t}}^{\mathrm{L}}+\mathrm{s}_{\mathrm{s}} \alpha_{\mathrm{Gs}} \mathrm{A}_{\mathrm{t}} \mathrm{K}_{\mathrm{e}, \mathrm{t}}^{\alpha_{\mathrm{e}}} \mathrm{K}_{\mathrm{s}, \mathrm{t}}^{\alpha_{\mathrm{s}}} \mathrm{G}_{\mathrm{s}, \mathrm{t}}^{\alpha_{\mathrm{Gs}}} \mathrm{G}_{\mathrm{t}}^{\alpha_{\mathrm{Gs}}} \mathrm{L}_{\mathrm{t}}^{\alpha_{3}}
\end{aligned}
$$

Siendo:

$$
\begin{gathered}
s=s_{e}+s_{s} \\
\alpha_{G}=\alpha_{G e}+\alpha_{G s}
\end{gathered}
$$

Los relacionados con los factores de producción mano de obra:

$$
W_{t}^{e} L_{t}=\alpha_{L} A_{t} K_{e, t}^{\alpha_{e}} K_{s, t}^{\alpha_{s}} G_{s, t}^{\alpha_{G s}} G_{t}^{\alpha_{G s}} L_{t}^{\alpha_{L}}+(1-s) \alpha_{G} A_{t} K_{e, t}^{\alpha_{e}} K_{s, t}^{\alpha_{s}} G_{s, t}^{\alpha_{G s}} G_{t}^{\alpha_{G s}} L_{t}^{\alpha_{L}}
$$

Como podemos comprobar, el retorno efectivo al capital $\left(\mathrm{R}_{\mathrm{Et}}^{\mathrm{e}}+\mathrm{R}_{\mathrm{St}}^{\mathrm{e}}\right)$; incluye la proporción, de la renta generada por los inputs públicos, siendo $\mathrm{S}=\mathrm{S}_{\mathrm{E}}+\mathrm{s}_{\mathrm{S}}$, y el retorno efectivo al factor trabajo $\mathrm{W}_{\mathrm{t}}^{\mathrm{e}}$; absorbe el porcentaje restante $(1-\mathrm{s})$ de la renta generada por los inputs públicos:

Sabiendo que:

$$
\begin{gathered}
{\left[\alpha_{e}+\alpha_{s}\right]+\alpha_{G}+\alpha_{L}=1} \\
{\left[\alpha_{e}+\alpha_{s}\right]+\left[\alpha_{G e}+\alpha_{G s}\right]+\alpha_{L}=1}
\end{gathered}
$$

Y siendo:

$$
\alpha_{1}=\alpha_{\mathrm{e}}+\alpha_{\mathrm{s}}
$$

Como hemos supuesto que:

$$
\begin{gathered}
s=s_{E}+s_{S} \\
s_{E}=\frac{\alpha_{e}}{\left(\alpha_{e}+\alpha_{s}+\alpha_{L}\right)} \\
s_{S}=\frac{\alpha_{s}}{\left(\alpha_{e}+\alpha_{s}+\alpha_{L}\right)}
\end{gathered}
$$

Dado que hemos dicho que:

$$
\begin{gathered}
\mathrm{R}_{\mathrm{et}}^{\mathrm{e}} \mathrm{K}_{\mathrm{et}}=\alpha_{\mathrm{e}} \mathrm{A}_{\mathrm{t}} \mathrm{K}_{\mathrm{e}, \mathrm{t}}^{\alpha_{\mathrm{e}}} \mathrm{K}_{\mathrm{s}, \mathrm{t}}^{\alpha_{\mathrm{s}}} \mathrm{G}_{\mathrm{s}, \mathrm{t}}^{\alpha_{G s}} \mathrm{G}_{\mathrm{t}}^{\alpha_{G s}} \mathrm{~L}_{\mathrm{t}}^{\alpha_{\mathrm{L}}}+\mathrm{s}_{\mathrm{e}} \alpha_{\mathrm{Ge}} \mathrm{A}_{\mathrm{t}} \mathrm{K}_{\mathrm{e}, \mathrm{t}}^{\alpha_{\mathrm{e}}} \mathrm{K}_{\mathrm{s}, \mathrm{t}}^{\alpha_{\mathrm{s}}} \mathrm{G}_{\mathrm{s}, \mathrm{t}}^{\alpha_{G s}} \mathrm{G}_{\mathrm{t}}^{\alpha_{\mathrm{Gs}}} \mathrm{L}_{\mathrm{t}}^{\alpha_{\mathrm{L}}} \\
\mathrm{R}_{\mathrm{et}}^{\mathrm{e}} \mathrm{K}_{\mathrm{st}}=\alpha_{\mathrm{e}}\left[\mathrm{Y}_{\mathrm{t}}\right]+\mathrm{s}_{\mathrm{e}} \alpha_{\mathrm{Ge}}\left[\mathrm{Y}_{\mathrm{t}}\right]
\end{gathered}
$$

Entonces se obtendría que:

$$
\begin{gathered}
\mathrm{R}_{\mathrm{et}}^{\mathrm{e}} \mathrm{K}_{\mathrm{et}}=\alpha_{\mathrm{e}}\left(1+\frac{\alpha_{\mathrm{Ge}}}{\left(\alpha_{\mathrm{e}}+\alpha_{\mathrm{s}}+\alpha_{\mathrm{L}}\right)}\right) \mathrm{Y}_{\mathrm{t}}=\left[\alpha_{\mathrm{M}}\right] \mathrm{Y}_{\mathrm{t}} \\
\alpha_{\mathrm{M}}=\alpha_{\mathrm{e}}\left(1+\frac{\alpha_{\mathrm{Ge}}}{\left(\alpha_{\mathrm{e}}+\alpha_{\mathrm{s}}+\alpha_{\mathrm{L}}\right)}\right) \\
\alpha_{\mathrm{M}}=\alpha_{\mathrm{e}}+\left(\frac{\alpha_{\mathrm{e}} \alpha_{\mathrm{Ge}}}{\left(\alpha_{\mathrm{e}}+\alpha_{\mathrm{s}}+\alpha_{\mathrm{L}}\right)}\right) \\
\mathrm{R}_{\mathrm{et}}^{\mathrm{e}}=\left[\alpha_{\mathrm{M}}\right] \frac{\mathrm{Y}_{\mathrm{t}}}{\mathrm{K}_{\mathrm{et}}} \\
{\left[\alpha_{\mathrm{M}}\right]=\alpha_{\mathrm{e}}+\mathrm{s}_{\mathrm{e}} \alpha_{\mathrm{Ge}}}
\end{gathered}
$$

Siendo:

$$
\alpha=\alpha_{M}+\alpha_{N}
$$

De igual modo, como:

$$
\begin{gathered}
\mathrm{R}_{\mathrm{St}}^{\mathrm{e}} \mathrm{K}_{\mathrm{st}}=\alpha_{\mathrm{S}} \mathrm{A}_{\mathrm{t}} \mathrm{K}_{\mathrm{e}, \mathrm{t}}^{\alpha_{\mathrm{e}}} \mathrm{K}_{\mathrm{s}, \mathrm{t}}^{\alpha_{\mathrm{s}}} \mathrm{G}_{\mathrm{s}, \mathrm{t}}^{\alpha_{G e}} \mathrm{G}_{\mathrm{t}}^{\alpha_{\mathrm{Gs}}} \mathrm{L}_{\mathrm{t}}^{\alpha_{\mathrm{L}}}+\mathrm{s}_{\mathrm{S}} \alpha_{\mathrm{Gs}} \mathrm{A}_{\mathrm{t}} \mathrm{K}_{\mathrm{e}, \mathrm{t}}^{\alpha_{\mathrm{e}}} \mathrm{K}_{\mathrm{s}, \mathrm{t}}^{\alpha_{\mathrm{s}}} \mathrm{G}_{\mathrm{t}}^{\alpha_{\mathrm{Ge}}} \mathrm{G}_{\mathrm{t}}^{\alpha_{\mathrm{Gs}}} \mathrm{L}_{\mathrm{t}}^{\alpha_{\mathrm{L}}} \\
\mathrm{R}_{\mathrm{St}}^{\mathrm{e}} \mathrm{K}_{\mathrm{st}}=\alpha_{\mathrm{S}}\left[\mathrm{Y}_{\mathrm{t}}\right]+\mathrm{s}_{\mathrm{S}} \alpha_{\mathrm{Gs}}\left[\mathrm{Y}_{\mathrm{t}}\right]
\end{gathered}
$$


se obtendría que:

$$
\begin{gathered}
\mathrm{R}_{\mathrm{st}}^{\mathrm{e}} \mathrm{K}_{\mathrm{st}}=\alpha_{\mathrm{s}}\left(1+\frac{\alpha_{\mathrm{Gs}}}{\left(\alpha_{\mathrm{e}}+\alpha_{\mathrm{s}}+\alpha_{\mathrm{L}}\right)}\right) \mathrm{Y}_{\mathrm{t}}=\left[\alpha_{\mathrm{N}}\right] \mathrm{Y}_{\mathrm{t}} \\
\alpha_{\mathrm{N}}=\alpha_{\mathrm{s}}\left(1+\frac{\alpha_{\mathrm{Gs}}}{\left(\alpha_{\mathrm{e}}+\alpha_{\mathrm{s}}+\alpha_{\mathrm{L}}\right)}\right) \\
\alpha_{\mathrm{N}}=\alpha_{\mathrm{s}}+\left(\frac{\alpha_{\mathrm{s}} \alpha_{\mathrm{Gs}}}{\left(\alpha_{\mathrm{e}}+\alpha_{\mathrm{s}}+\alpha_{\mathrm{L}}\right)}\right) \\
\mathrm{R}_{\mathrm{st}}^{\mathrm{e}}=\left[\alpha_{\mathrm{N}}\right] \frac{\mathrm{Y}_{\mathrm{t}}}{\mathrm{K}_{\mathrm{st}}} \\
{\left[\alpha_{\mathrm{N}}\right]=\alpha_{\mathrm{s}}+\mathrm{s}_{\mathrm{s}} \alpha_{\mathrm{Gs}}}
\end{gathered}
$$

Siendo:

$$
\alpha=\alpha_{M}+\alpha_{N}
$$

$$
\begin{gathered}
\mathrm{W}_{\mathrm{t}}^{\mathrm{e}} \mathrm{L}_{\mathrm{t}}=\alpha_{3}\left(1+\frac{\alpha_{\mathrm{G}}}{\left(\alpha_{\mathrm{e}}+\alpha_{\mathrm{s}}+\alpha_{3}\right)}\right) \mathrm{Y}_{\mathrm{t}}=[1-\alpha] \mathrm{Y}_{\mathrm{t}} \\
\mathrm{W}_{\mathrm{t}}^{\mathrm{e}}=[1-\alpha] \frac{\mathrm{Y}_{\mathrm{t}}}{\mathrm{L}_{\mathrm{t}}} \\
\mathrm{W}_{\mathrm{t}}^{\mathrm{e}}=\left[1-\left(\alpha_{\mathrm{M}}+\alpha_{\mathrm{N}}\right)\right] \frac{\mathrm{Y}_{\mathrm{t}}}{\mathrm{L}_{\mathrm{t}}}
\end{gathered}
$$

donde $\alpha$ es la participación de las rentas del capital (privado) en la renta total, y como se ha indicado:

$$
\alpha=\alpha_{M}+\alpha_{N}
$$

y $(1-\alpha)$ es la participación de las rentas del trabajo en la renta total.

Vemos que se verifica:

$$
\begin{gathered}
\alpha+(1-\alpha)=1 \\
\alpha_{M}+\alpha_{N}+(1-\alpha)=1
\end{gathered}
$$

La relación entre los ingresos de los factores privados y las rentas generadas por el capital público sería:

$$
\begin{aligned}
\frac{\mathrm{R}_{\mathrm{Et}}^{\mathrm{e}} \mathrm{K}_{\mathrm{et}}}{\frac{\partial \mathrm{Y}_{\mathrm{t}}}{\partial \mathrm{G}_{\mathrm{et}}} \mathrm{G}_{\mathrm{et}}} & =\frac{\alpha_{\mathrm{E}}\left(1+\begin{array}{c}
\alpha_{\mathrm{Ge}} \\
\left(\alpha_{\mathrm{e}}+\alpha_{\mathrm{s}}+\alpha_{\mathrm{L}}\right)
\end{array}\right)}{\alpha_{\mathrm{Ge}}} \\
\frac{\mathrm{R}_{\mathrm{St}}^{\mathrm{e}} \mathrm{K}_{\mathrm{St}}}{\frac{\partial \mathrm{Y}_{\mathrm{t}}}{\partial \mathrm{G}_{\mathrm{st}}} \mathrm{G}_{\mathrm{st}}} & =\frac{\alpha_{\mathrm{S}}\left(1+\frac{\alpha_{\mathrm{Gs}}}{\left(\alpha_{\mathrm{e}}+\alpha_{\mathrm{s}}+\alpha_{\mathrm{L}}\right)}\right)}{\alpha_{\mathrm{Gs}}} \\
\frac{\mathrm{W}_{\mathrm{t}}^{\mathrm{e}} \mathrm{L}_{\mathrm{t}}}{\partial \mathrm{Y}_{\mathrm{t}}} & \left.=\frac{\alpha_{3}\left(1+\frac{\alpha_{\mathrm{G}}}{\partial \mathrm{G}_{\mathrm{t}}} \mathrm{G}_{\mathrm{t}}\right.}{\left.\alpha_{\mathrm{G}}+\alpha_{\mathrm{s}}+\alpha_{\mathrm{L}}\right)}\right)
\end{aligned}
$$

Finalmente, la economía debe cumplir la siguiente restricción de factibilidad:

$$
\mathrm{C}_{\mathrm{t}}+\left[\mathrm{I}_{\mathrm{e}, \mathrm{t}}+\mathrm{I}_{\mathrm{s}, \mathrm{t}}\right]+\left[\mathrm{I}_{\mathrm{Ge}, \mathrm{t}}+\mathrm{I}_{\mathrm{Gs}, \mathrm{t}}\right]=\left[\mathrm{R}_{\mathrm{et}}^{\mathrm{e}} \mathrm{K}_{\mathrm{et}}+\mathrm{R}_{\mathrm{st}}^{\mathrm{e}} \mathrm{K}_{\mathrm{st}}\right]+\mathrm{W}_{\mathrm{t}}^{\mathrm{e}} \mathrm{L}_{\mathrm{t}}
$$

o lo que es lo mismo:

$$
\mathrm{C}_{\mathrm{t}}+\left[\mathrm{I}_{\mathrm{e}, \mathrm{t}}+\mathrm{I}_{\mathrm{s}, \mathrm{t}}\right]+\left[\mathrm{I}_{\mathrm{Ge}, \mathrm{t}}+\mathrm{I}_{\mathrm{Gs}, \mathrm{t}}\right]=\mathrm{Y}_{\mathrm{t}}
$$

En efecto, podemos comprobar que la citada restricción de factibilidad de la economía es la suma de las siguientes restricciones consideradas en el modelo:

La restricción de los consumidores:

$$
\left(1+\tau_{t}^{\mathrm{e}}\right) \mathrm{C}_{\mathrm{t}}+\left[\mathrm{I}_{\mathrm{e}, \mathrm{t}}+\mathrm{I}_{\mathrm{s}, \mathrm{t}}\right]=\left(1-\tau_{\mathrm{t}}^{\mathrm{l}}\right) \mathrm{W}_{\mathrm{t}}^{\mathrm{e}} \mathrm{L}_{\mathrm{t}}+\left(1-\tau_{\mathrm{t}}^{\mathrm{k}}\right)\left[\mathrm{R}_{\mathrm{Et}}^{\mathrm{e}} \mathrm{K}_{\mathrm{e}, \mathrm{t}}+\mathrm{R}_{\mathrm{St}}^{\mathrm{e}} \mathrm{K}_{\mathrm{s}, \mathrm{t}}\right]+\mathrm{T}_{\mathrm{t}}
$$


La restricción del gobierno:

$$
\left[\mathrm{I}_{\mathrm{Ge}, \mathrm{t}}+\mathrm{I}_{\mathrm{Gs}, \mathrm{t}}\right]+\mathrm{T}_{\mathrm{t}}=\tau_{\mathrm{t}}^{\mathrm{c}} \mathrm{C}_{\mathrm{t}}+\tau_{\mathrm{t}}^{\mathrm{l}} \mathrm{W}_{\mathrm{t}}^{\mathrm{e}} \mathrm{L}_{\mathrm{t}}+\tau_{\mathrm{t}}^{\mathrm{k}}\left[\left(\mathrm{R}_{\mathrm{et}}^{\mathrm{e}}-\delta_{\mathrm{Ke}}\right) \mathrm{K}_{\mathrm{et}}+\tau_{\mathrm{t}}^{\mathrm{k}}\left(\mathrm{R}_{\mathrm{st}}^{\mathrm{e}}-\delta_{\mathrm{Ks}}\right) \mathrm{K}_{\mathrm{st}}\right]
$$

\subsection{El equilibrio del modelo}

El equilibrio de la economía lo obtenemos combinando las condiciones de primer orden del consumidor con las condiciones de primer orden del problema de maximización de beneficios de la empresa, del modo que, de (6.14) y de (6.19), obtenemos:
Las FOC del consumidor

(22) $\frac{1}{1-\mathrm{L}_{\mathrm{t}}}=\frac{\gamma}{(1-\gamma)} \frac{\left(1+\tau_{\mathrm{t}}^{\mathrm{l}}\right)}{\left(1+\tau_{\mathrm{t}}^{\mathrm{e}}\right)} \frac{\mathrm{W}_{\mathrm{t}}^{\mathrm{e}}}{\mathrm{C}_{\mathrm{t}}}$

De (6.15) y de (6.20), obtenemos

$$
\frac{\left(1+\tau_{t}^{c}\right)}{\left(1+\tau_{t-1}^{c}\right)} \frac{C_{t}}{C_{t-1}} \frac{1}{\beta}=\frac{Z_{t-1}}{Z_{t}}\left[\left(1-\tau_{t}^{k}\right)\left(Z_{t} R_{E t}^{e}+\delta_{K e}\right)+1\right]
$$

De (16) y de (21), obtenemos

$$
\left.\frac{\left(1+\tau_{t}^{\mathrm{c}}\right)}{\left(1+\tau_{\mathrm{t}-1}^{\mathrm{c}}\right)} \frac{\mathrm{C}_{\mathrm{t}}}{\mathrm{C}_{\mathrm{t}-1}} \frac{1}{\beta}=\left[\left(1-\tau_{\mathrm{t}}^{\mathrm{k}}\right) \mathrm{R}_{\mathrm{st}}^{\mathrm{e}}+\delta_{\mathrm{Ks}}\right)+1\right]
$$

Para cerrar el modelo, la economía debe cumplir la siguiente condición de factibilidad: (25)

$$
\begin{gathered}
\quad\left(1+\tau_{t}^{\mathrm{e}}\right) \mathrm{C}_{\mathrm{t}}+\frac{\mathrm{K}_{\mathrm{e}, \mathrm{t}+1}}{\mathrm{Z}_{\mathrm{t}}}-\frac{\mathrm{K}_{\mathrm{e}, \mathrm{t}}}{\mathrm{Z}_{\mathrm{t}}}+\mathrm{K}_{\mathrm{s}, \mathrm{t}+1}-\mathrm{K}_{\mathrm{s}, \mathrm{t}}+\mathrm{I}_{\mathrm{G}, \mathrm{t}} \\
=\left(1-\tau_{\mathrm{t}}^{\mathrm{l}}\right) \mathrm{W}_{\mathrm{t}}^{\mathrm{e}} \mathrm{L}_{\mathrm{t}_{\mathrm{t}}}+\left(1-\tau_{\mathrm{t}}^{\mathrm{k}}\right) \frac{1}{\mathrm{Z}_{\mathrm{t}}}\left(\mathrm{Z}_{\mathrm{t}} \mathrm{R}_{\mathrm{Et}}^{\mathrm{e}}+\delta_{\mathrm{Ke}}\right) \mathrm{K}_{\mathrm{e}, \mathrm{t}}+\left(1-\tau_{\mathrm{t}}^{\mathrm{k}}\right)\left(\mathrm{R}_{\mathrm{St}}^{\mathrm{e}}+\delta_{\mathrm{Ks}}\right) \mathrm{K}_{\mathrm{s}, \mathrm{t}} \\
+\mathrm{T}_{\mathrm{t}} \\
\mathrm{C}_{\mathrm{t}}+\left[\mathrm{I}_{\mathrm{e}, \mathrm{t}}+\mathrm{I}_{\mathrm{s}, \mathrm{t}}\right]+\left[\mathrm{I}_{\mathrm{Ge}, \mathrm{t}}+\mathrm{I}_{\mathrm{Gs}, \mathrm{t}}\right]=\mathrm{Y}_{\mathrm{t}}
\end{gathered}
$$

FOC de las empresas

$$
\begin{aligned}
& W_{t}=\alpha_{L} A_{t} K_{e, t}^{\alpha_{e}} K_{s, t}^{\alpha_{s}} G_{e, t}^{\alpha_{G e}} G_{s t}^{\alpha_{G s}} L_{t}^{\alpha_{L}-1} \\
& R_{e t}=\alpha_{e} A_{t} K_{e, t}^{\alpha_{e}-1} K_{s, t}^{\alpha_{s}} G_{e, t}^{\alpha_{G e}} G_{t s}^{\alpha_{G s}} L_{t}^{\alpha_{L}} \\
& \mathrm{R}_{\mathrm{st}}=\alpha_{\mathrm{s}} \mathrm{A}_{\mathrm{t}} \mathrm{K}_{\mathrm{e}, \mathrm{t}}^{\alpha_{\mathrm{e}}} \mathrm{K}_{\mathrm{s}, \mathrm{t}}^{\alpha_{\mathrm{s}}-1} \mathrm{G}_{\mathrm{e}, \mathrm{t}}^{\alpha_{\mathrm{Ge}}} \mathrm{G}_{\mathrm{st}}^{\alpha_{\mathrm{Gs}}} \mathrm{L}_{\mathrm{t}}^{\alpha_{\mathrm{L}}} \\
& \mathrm{R}_{\mathrm{et}}^{\mathrm{e}} \mathrm{K}_{\mathrm{et}}=\alpha_{\mathrm{e}} \mathrm{A}_{\mathrm{t}} \mathrm{K}_{\mathrm{e}, \mathrm{t}}^{\alpha_{\mathrm{e}}} \mathrm{K}_{\mathrm{s}, \mathrm{t}}^{\alpha_{\mathrm{s}}} \mathrm{G}_{\mathrm{s}, \mathrm{t}}^{\alpha_{\mathrm{Gs}}} \mathrm{G}_{\mathrm{t}}^{\alpha_{\mathrm{Gs}}} \mathrm{L}_{\mathrm{t}}^{\alpha_{\mathrm{L}}}+\mathrm{s}_{\mathrm{e}} \alpha_{\mathrm{Ge}} \mathrm{A}_{\mathrm{t}} \mathrm{K}_{\mathrm{e}, \mathrm{t}}^{\alpha_{\mathrm{e}}} \mathrm{K}_{\mathrm{s}, \mathrm{t}}^{\alpha_{s}} \mathrm{G}_{\mathrm{s}, \mathrm{t}}^{\alpha_{\mathrm{Gs}}} \mathrm{G}_{\mathrm{t}}^{\alpha_{\mathrm{Gs}}} \mathrm{L}_{\mathrm{t}}^{\alpha_{\mathrm{L}}} \\
& \mathrm{R}_{\mathrm{Et}}^{\mathrm{e}}=\mathrm{R}_{\mathrm{et}}+\mathrm{s}_{\mathrm{e}} \alpha_{\mathrm{Ge}} \mathrm{A}_{\mathrm{t}} \mathrm{K}_{\mathrm{e}, \mathrm{t}}^{\alpha_{\mathrm{e}}-1} \mathrm{~K}_{\mathrm{s}, \mathrm{t}}^{\alpha_{\mathrm{s}}} \mathrm{G}_{\mathrm{e}, \mathrm{t}}^{\alpha_{\mathrm{Ge}}} \mathrm{G}_{\mathrm{s}, \mathrm{t}}^{\alpha_{\mathrm{Gs}}} \mathrm{L}_{\mathrm{t}}^{\alpha_{\mathrm{L}}} \\
& \mathrm{R}_{\mathrm{St}}^{\mathrm{e}} \mathrm{K}_{\mathrm{st}}=\alpha_{\mathrm{S}} \mathrm{A}_{\mathrm{t}} \mathrm{K}_{\mathrm{e}, \mathrm{t}}^{\alpha_{\mathrm{e}}} \mathrm{K}_{\mathrm{s}, \mathrm{t}}^{\alpha_{\mathrm{s}}} \mathrm{G}_{\mathrm{s}, \mathrm{t}}^{\alpha_{\mathrm{Ge}}} \mathrm{G}_{\mathrm{t}}^{\alpha_{\mathrm{Gs}}} \mathrm{L}_{\mathrm{t}}^{\alpha_{\mathrm{L}}}+\mathrm{s}_{\mathrm{S}} \alpha_{\mathrm{Gs}} \mathrm{A}_{\mathrm{t}} \mathrm{K}_{\mathrm{e}, \mathrm{t}}^{\alpha_{\mathrm{e}}} \mathrm{K}_{\mathrm{s}, \mathrm{t}}^{\alpha_{\mathrm{s}}} \mathrm{G}_{\mathrm{t}}^{\alpha_{\mathrm{Ge}}} \mathrm{G}_{\mathrm{t}}^{\alpha_{\mathrm{Gs}}} \mathrm{L}_{\mathrm{t}}^{\alpha_{\mathrm{L}}} \\
& \mathrm{R}_{\mathrm{st}}^{\mathrm{e}}=\mathrm{R}_{\mathrm{st}}+\mathrm{s}_{\mathrm{s}} \alpha_{\mathrm{Gs}} \mathrm{A}_{\mathrm{t}} \mathrm{K}_{\mathrm{e}, \mathrm{t}}^{\alpha_{\mathrm{e}}} \mathrm{K}_{\mathrm{s}, \mathrm{t}}^{\alpha_{s-1}} \mathrm{G}_{\mathrm{e}, \mathrm{t}}^{\alpha_{\mathrm{Ge}}} \mathrm{G}_{\mathrm{s}, \mathrm{t}}^{\alpha_{\mathrm{Gs}}} \mathrm{L}_{\mathrm{t}}^{\alpha_{\mathrm{L}}} \\
& W_{t}^{e}=W_{t}+(1-s) \alpha_{G} A_{t} K_{e, t}^{\alpha_{e}} K_{s, t}^{\alpha_{s}} G_{e, t}^{\alpha_{G e}} G_{s, t}^{\alpha_{G s}} L_{t}^{\alpha_{L}-1}
\end{aligned}
$$


Siendo:

$$
\mathrm{s}=\mathrm{s}_{\mathrm{e}}+\mathrm{s}_{\mathrm{s}}
$$

y la condición de factibilidad de la economía (25), caracterizan el equilibrio competitivo de la economía.

\subsection{Ecuaciones del modelo y calibración}

Tenemos un modelo con tres perturbaciones: la perturbación de productividad total de la economía, una nueva perturbación que refleja el cambio tecnológico asociado a la inversión (la productividad específica en la inversión privada) $Z_{t}$, y la eventual actuación del gobierno para la adquisiciones de sus inversiones públicas, $B_{t}$, tanto las directamente asociadas a las infraestructuras, como las asociadas al progreso tecnológico de la inversión privada, cuando se realiza como proporción de la misma.

El equilibrio competitivo del modelo viene dado por un conjunto de diecisiete ecuaciones que representan el comportamiento de las catorce variables endógenas,

$$
Y_{t}, C_{t}, I_{e t}, I_{s t}, I_{G e t}, I_{G s t}, K_{e t}, K_{s t}, G_{e t}, G_{s t}, L_{t}, R_{e t}, R_{s t}, W_{t}
$$

y de las seis variables exógenas

$$
A_{t}, B_{t} Z_{t}, \tau_{t}^{c}, \tau_{t}^{l}, \tau_{t}^{k}
$$

Y con las FOC antes señaladas, para los consumidores, para las empresas, y para la condición de equilibrio del gobierno, así como las condiciones generales que debe cumplir la economía unidas a las perturbaciones estocásticas citadas, obtenemos un sistema de 20 ecuaciones, correspondientes a las referidas variables endógenas citadas.

Como se ha dicho, en el modelo desarrollado tenemos tres perturbaciones tecnológicas, una neutral y otras dos específicas, que representan el

$$
\left[\begin{array}{l}
\ln A_{t} \\
\ln B_{t} \\
\ln Z_{t}
\end{array}\right]=\left[\begin{array}{ccc}
\rho_{A} & v_{A B} & v_{A Z} \\
v_{B A} & \rho_{B} & v_{B Z} \\
v_{Z A} & v_{Z B} & \rho_{Z}
\end{array}\right]\left[\begin{array}{l}
\ln A_{t-1} \\
\ln B_{t-1} \\
\ln Z_{t-1}
\end{array}\right]+\left[\begin{array}{c}
\varepsilon_{t}^{A} \\
\varepsilon_{t}^{B} \\
\varepsilon_{t}^{Z}
\end{array}\right]
$$

Y donde: $\left|\rho_{\mathrm{i}} \pm \mathrm{v}\right|<1, \mathrm{i}=\mathrm{A}, \mathrm{B}, \mathrm{Z}$; con el objeto de asegurar la estacionariedad de los procesos, siendo

$$
\begin{aligned}
& E\left(\varepsilon_{t}^{i}\right)=0, E\left(\varepsilon_{t}^{i}, \varepsilon_{t}^{i}\right)=\sigma_{i}^{2}, \forall i \\
& \Omega=\left\{\alpha_{M}, \alpha_{N}, \alpha_{E}, \alpha_{S}, \alpha_{L}, \alpha_{G e}, \alpha_{G s}, \beta, \gamma, \delta_{G E}, \delta_{G S}, \delta_{E}, \delta_{S}, \rho_{A}, \sigma_{A}, \rho_{B}, \sigma_{B}, \rho_{Z}, \sigma_{Z}\right\}
\end{aligned}
$$

Los valores asignados a parámetros que aparecen en este modelo son los que se muestran en la Tabla 2. Estos parámetros se han obtenido de Torres (2009) y de Jiménez Bastida (2016) y 
Tabla 2. Parámetros calibrados para el MODELO J

\begin{tabular}{|c|l|c|}
\hline Parámetro & \multicolumn{1}{|c|}{ Definición } & Valor \\
\hline$\alpha_{\mathrm{M}}$ & $\alpha_{\mathrm{M}}=\alpha_{\mathrm{e}}+\alpha_{\mathrm{e}} \alpha_{\mathrm{GE}} /\left(\alpha_{\mathrm{e}}+\alpha_{\mathrm{s}}+\alpha_{\mathrm{L}}\right)$ & 0.2138 \\
\hline$\alpha_{\mathrm{N}}$ & $\alpha_{\mathrm{N}}=\alpha_{\mathrm{s}}+\alpha_{\mathrm{s}} \alpha_{\mathrm{GS}} /\left(\alpha_{\mathrm{e}}+\alpha_{\mathrm{s}}+\alpha_{\mathrm{L}}\right)$ & 0.15465 \\
\hline$\alpha_{\mathrm{e}}$ & Proporción rentas capital en equipos s/renta total & 0.205 \\
\hline$\alpha_{\mathrm{s}}$ & Proporción rentas capital en estructuras s/renta total & 0.110 \\
\hline$\alpha_{\mathrm{GS}}$ & Proporción rentas capital público INFRAESTRUCTURAS s/renta total & 0.0310 \\
\hline$\alpha_{\mathrm{GE}}$ & Proporción rentas capital público EQUIPOS Y SISTEMAS s/renta total & 0.0690 \\
\hline$\alpha_{\mathrm{L}}$ & Proporción rentas del trabajo s/renta total & 0.585 \\
\hline$\beta$ & Factor de descuento & 0.970 \\
\hline$\gamma$ & Parámetro de preferencias & 0.45 \\
\hline$\delta_{\mathrm{e}}$ & Parámetro de depreciación equipos privados & 0.06 \\
\hline$\delta_{\mathrm{s}}$ & Parámetro de depreciación estructuras privadas & 0.02 \\
\hline$\delta_{\mathrm{Ge}}$ & Parámetro de depreciación equipos y sistemas públicos & 0.08 \\
\hline$\delta_{\mathrm{Gs}}$ & Parámetro de depreciación estructuras públicos & 0.02 \\
\hline$\rho_{\mathrm{A}}$ & Parámetro autorregresivo Productividad Total Fact. (PTF) & 0.95 \\
\hline$\sigma_{\mathrm{A}}$ & Desviación estándar PTF & 0.001 \\
\hline$\rho_{\mathrm{B}}$ & Parámetro autorregresivo proporción GASTO PUBLICO & 0.95 \\
\hline$\sigma_{\mathrm{B}}$ & Desviación estándar proporción GASTO PUBLICO & 0.001 \\
\hline$\rho_{\mathrm{Z}}$ & Parámetro autorregresivo Compont. Tecnol. Espc.(TICs) & 0.95 \\
\hline$\sigma_{\mathrm{Z}}$ & Desviación estándar CTE (TICs) & 0.001 \\
\hline & & \\
\hline
\end{tabular}

se utilizarán para simular el MODELO J. Se considera que los parámetros que definen el proceso estocástico para la tecnología específica a los nuevos activos en bienes de equipo es igual al proceso estocástico de la productividad total de los factores (PTF), valores que son muy similares a los que aparecen en la literatura.

Como indica Torres (2013), en Greenwood et al. (2000), y para la economía de Estados Unidos se estima un valor del parámetro autorregresivo de 0,64 para el cambio tecnológico específico. Pakko (2005) usando un modelo similar estima, también para Estados Unidos, un parámetro de 0,945 para el cambio tecnológico neutral y de 0,941 para el cambio tecnológico específico. Rodríguez y Torres (2009) estiman valores de estos parámetros, en el caso de la productividad agregada, de 0,95 para Estados Unidos, 0,83 para Japón y de 0,72 para Alemania, con una desviación estándar de 0,0130,014 .

En el caso de los activos TIC, los valores son 0,97 ; 0,96; y 0,85, para Estados Unidos,
Japón y Alemania, respectivamente. Por lo que se refiere a los activos no-TIC, los valores son $0,92,0,89$ y 0,81 , para los tres países, respectivamente.

\subsection{Determinación del estado estacionario del modelo $\mathrm{J}$}

Una vez obtenido el equilibrio de la economía, se procede a definir los valores de estado estacionario. Se dice que el modelo presentado anteriormente es estacionario, cuando se puede verificar que existe un valor para las variables que se mantiene constante en el tiempo. Para calcular el estado estacionario, en primer lugar, se procede a eliminar los subíndices de tiempo de las variables. Así, recordando que la definición de estado estacionario indica una situación en la cual las variables se mantienen constantes periodo a periodo, esto significa, que por ejemplo, para el consumo, que se tendría:

$$
\ldots=C_{t-1}=C_{t}=C_{t+1}=\cdots=\bar{C}
$$


Y lo mismo se haría para todas las variables consideradas en el modelo. Por tanto, el modelo obtenido del equilibrio de la economía se puede transformar para cada una de las variables indicadas. Una vez obtenidas las condiciones de equilibrio, se introducen en Dynare, y se continúa con el proceso de resolución del modelo?.

Una vez calibrado el Modelo J, y obtenido el estado estacionario del mismo, se procede a resolver el problema de optimización dinámica de los agentes, sometidos a sus respectivas restricciones presupuestarias. Además, sirve para calcular los efectos de una perturbación de productividad total de los factores sobre la economía modelizada, $A_{t}$, así como de la perturbación que refleja el cambio tecnológico asociado a la inversión $Z_{t}$ (La productividad específica en la inversión privada), y la eventual actuación del gasto del gobierno para las correspondientes adquisiciones de sus inversiones públicas ${ }^{10} B_{t}$.

Entre alguno de los resultados destacables de la resolución del modelo, más allá de las funciones irf, ante las tres perturbaciones introducidas, y que se analizaran con detenimiento en el apartado siguiente, se muestran en la siguiente Tabla 3, las funciones que generan la dinámica de las variables decisión y las de las funciones de transición.

Tabla 3. Funciones de decisión y de transición Modelo J

\begin{tabular}{|c|c|c|c|c|c|c|c|c|c|c|c|c|c|c|}
\hline & $Y$ & C & IE & IS & $\mathrm{KE}$ & KS & L & w & IGE & IGS & GE & GS & $\mathrm{RE}$ & RS \\
\hline Constant & 0.175510 & 0.153837 & 0.016458 & 0.004776 & 0.279454 & 0.256976 & 0.260109 & 0.308137 & 0.000263 & 0.000176 & 0.003289 & 0.008771 & 0.100554 & -0.002430 \\
\hline (correction) & 0.000100 & -0.000114 & 0.000137 & 0.000077 & 0.000138 & 0.000077 & 0.000252 & -0.000124 & 0 & 0 & 0 & 0 & 0.000057 & -0.000001 \\
\hline KE(-1) & -0.174590 & 0.459450 & -0.855504 & 0.221900 & 0.084060 & 0.221463 & -0.765424 & 0.603063 & -0.000262 & -0.000175 & -0.000262 & -0.000175 & -0.459212 & 0.002403 \\
\hline $\mathrm{KS}(-1)$ & -0.251441 & 0.438110 & 0.079889 & -0.768811 & 0.079260 & 0.210560 & -0.823029 & 0.537171 & -0.000377 & -0.000251 & -0.000377 & -0.000251 & -0.143174 & 0.010950 \\
\hline $\mathrm{GE}(-1)$ & 7.305 .427 & -1.442 .918 & 5.489 .908 & 3.240 .174 & 5.508 .171 & 3.258 .437 & 9.812 .810 & 8.103 .410 & 0.010958 & 0.007305 & 0.930958 & 0.007305 & 6.436 .844 & -0.155564 \\
\hline $\mathrm{GS}(-1)$ & 1.440 .862 & -0.406510 & 1.155 .659 & 0.688111 & 1.159 .261 & 0.691713 & 2.087 .714 & $-1,027.786$ & 0.002161 & 0.001441 & 0.002161 & 0.981441 & 0.472332 & -0.011415 \\
\hline$A(-1)$ & 0.376186 & -0.089907 & 0.292124 & 0.173028 & 0.293065 & 0.173969 & 0.524797 & 0.034489 & 0.000564 & 0.000376 & 0.000564 & 0.000376 & 0.214204 & -0.00517 \\
\hline$z(-1)$ & 0.120919 & -0.133744 & 0.278054 & -0.023943 & 0.294527 & -0.023391 & 0.299671 & -0.147529 & 0.000431 & 0.000121 & 0.000681 & 0.000121 & 0.067799 & -0.001639 \\
\hline$B(-1)$ & 0.005057 & -0.003676 & 0.005244 & 0.003060 & 0.005673 & 0.003489 & 0.010138 & -0.004991 & 0.000258 & 0.000172 & 0.000258 & 0.000172 & 0.002294 & -0.000055 \\
\hline e & 0.395985 & -0.094639 & 0.307499 & 0.182135 & 0.308489 & 0.183125 & 0.552417 & 0.036304 & 0.000594 & 0.000396 & 0.000594 & 0.000396 & 0.225478 & -0.005449 \\
\hline u & 0.127283 & -0.140784 & 0.292688 & -0.025203 & 0.310029 & -0.024622 & 0.315443 & -0.155293 & 0.000454 & 0.000127 & 0.000717 & 0.000127 & 0.071367 & -0.001725 \\
\hline a & 0.005324 & -0.003869 & 0.005520 & 0.003221 & 0.005972 & 0.003673 & 0.010671 & -0.005253 & 0.000271 & 0.000181 & 0.000271 & 0.000181 & 0.002414 & -0.000058 \\
\hline tauc & -0.047811 & -0.083593 & 0.009778 & 0.026123 & 0.009659 & 0.026004 & -0.120331 & 0.059239 & -0.000072 & -0.000048 & -0.000072 & -0.000048 & -0.027224 & 0.000658 \\
\hline taul & -0.115095 & -0.067794 & -0.013600 & -0.033413 & -0.013888 & -0.033701 & -0.289673 & 0.142607 & -0.000173 & -0.000115 & -0.000173 & -0.000115 & -0.065537 & 0.001584 \\
\hline
\end{tabular}

Así mismo, en la Tabla 4, se muestran los datos estadísticos más significativos de las variables del modelo J.

Tabla 4. Principales datos estadísticos de las variables simuladas

\begin{tabular}{|c|c|c|c|c|c|}
\hline VARIABLE & MEAN & STD. DEV. & VARIANCE & SKEWNESS & KURTOSIS \\
\hline Y & 0.176405 & 0.010461 & 0.000109 & 0.231849 & -0.328415 \\
\hline C & 0.154816 & 0.009094 & 0.000083 & 0.222135 & -0.363868 \\
\hline IE & 0.016408 & 0.004485 & 0.000020 & 0.186618 & 0.219650 \\
\hline IS & 0.004736 & 0.002317 & 0.000005 & 0.184015 & 0.239920 \\
\hline KE & 0.283021 & 0.020257 & 0.000410 & 0.366795 & -0.123590 \\
\hline KS & 0.258232 & 0.013154 & 0.000173 & 0.221455 & -0.394681 \\
\hline L & 0.259937 & 0.006968 & 0.000049 & 0.214059 & 0.378596 \\
\hline W & 0.310477 & 0.018787 & 0.000353 & 0.232658 & -0.374706 \\
\hline IGE & 0.000268 & 0.000024 & 0.000000 & 0.558531 & 0.685362 \\
\hline IGS & 0.000177 & 0.000012 & 0.000000 & 0.503449 & 0.600499 \\
\hline GE & 0.003375 & 0.000304 & 0.000000 & 0.384048 & 0.040096 \\
\hline GS & 0.008822 & 0.000324 & 0.000000 & 0.272339 & 0.058776 \\
\hline RE & 0.100039 & 0.003548 & 0.000013 & 0.065174 & -0.037553 \\
\hline RS & -0.002434 & 0.000076 & 0.000000 & -0.156771 & -0.180458 \\
\hline A & 1.000 .313 & 0.034122 & 0.001164 & 0.172433 & -0.468452 \\
\hline Z & 1.007 .398 & 0.032352 & 0.001047 & 0.336820 & 0.148530 \\
\hline B & 1.003 .553 & 0.032771 & 0.001074 & -0.094233 & -0.720393 \\
\hline
\end{tabular}

9 De las 20 variables para las cuales hay que calcular su valor en estado estacionario en base a los parámetros calibrados, y modo de ejemplo, se muestra cómo queda en el estado estacionario, la expresión del factor de producción trabajo, en función de los parámetros del modelo.

$$
\overline{\mathrm{L}}==\frac{\frac{\gamma}{(1-\gamma)} \frac{\left(1-\bar{\tau}^{\mathrm{l}}\right)}{\left(1+\bar{\tau}^{\mathrm{c}}\right)}}{\left[\left[1-\delta_{\mathrm{KE}} \frac{\overline{\mathrm{Z}}\left(1-\bar{\tau}^{\mathrm{k}}\right) \alpha_{\mathrm{M}}}{\left[\frac{1}{\beta}-\left(1-\delta_{\mathrm{KE}}\right)\right]}-\delta_{\mathrm{KS}} \frac{\left(1-\bar{\tau}^{\mathrm{k}}\right) \alpha_{\mathrm{N}}}{\left[\frac{1}{\beta}-\left(1-\delta_{\mathrm{KS}}\right)\right]}-\overline{\mathrm{Z}} \theta-\overline{\mathrm{B}} \theta\right]+\left[\frac{\gamma}{(1-\gamma)} \frac{\left(1-\bar{\tau}^{\mathrm{l}}\right)}{\left(1+\bar{\tau}^{\mathrm{c}}\right)}\left[1-\left[\alpha_{\mathrm{M}}+\alpha_{\mathrm{N}}\right]\right]\right]\right.}
$$

10 Las ecuaciones que se introducen en el modelo y que utiliza Matlab/Dynare para su resolución son las que se muestran en la siguiente Tabla A1 del Anexo 
4. Funciones impulso respuesta resultado de perturbaciones introducidas en el modelo j: análisis macroeconómico de la economía modelizada

Como ya se ha dicho, una vez calibrado nuestro Modelo J, y obtenido el estado estacionario del mismo, se utiliza Matlab/Dynare para calcular la perturbación de productividad total de la economía, la perturbación que refleja el cambio tecnológico asociado a la inversión ( $\mathrm{La}$ productividad específica en la inversión privada), y la eventual actuación del gobierno para la adquisición de sus inversiones públicas .

Las funciones impulso respuesta (irf) asociadas a las respectivas perturbaciones estocásticas, y que inciden en cada una de las variables del modelo planteado, producen unos efectos muy singulares para cada una de las variables, que requiere un minucioso análisis y consideración. No obstante, los anterior, ya la mera visualización gráfica de los efectos que producen resultan muy interesantes, por lo novedoso del hallazgo y lo diferente a los planteamientos tradicionales efectuados al respecto. En ese sentido, la evolución de las variables del modelo ante las perturbaciones señaladas se muestra en las Figuras A1, A2 y A3 del Anexo, respectivamente. Un análisis comparado de las perturbaciones incluidas en el modelo se muestra en la Tabla 5.

A partir de ella se puede realizar las siguientes consideraciones:

- En primer lugar, la producción agregada (Y), ante una perturbación provocada por un aumento del $1 \%$ de la desviación estándar de las tres variables se comporta de manera positiva respecto a su nivel del estado estacionario, que, de forma paulatina, y transcurridos más de cuarenta periodos después, retoma los valores que tenía en su estado estacionario. En definitiva, mantiene un efecto de largo plazo acusado pero decreciente ante el shock.

- El consumo (C), tras las perturbaciones y una ligera disminución en los instantes iniciales, muestra una desviación positiva respecto a su valor en el nivel de equilibrio que, si bien es moderada, se mantiene por encima de dicho nivel.

- Con relación a la inversión privada en bienes de equipo (IE), experimenta un incremento positivo en el corto plazo, para posteriormente, trascurridos un reducido número de periodos converger a su nivel de equilibrio de partida. Este resultado es válido cuando la perturbación proviene tanto de la PTF, como del gasto público asociado a infraestructuras y bienes de equipo. Sin embargo, en el caso del progreso tecnológico asociado a la inversión privada, su efecto es mucho más amplio en el tiempo, volviendo al estado estacionario en el muy largo plazo. Este resultado avala claramente los planteamientos establecidos en las hipótesis de partida.

- La inversión privada en infraestructuras (IS), muestra un comportamiento similar a la inversión privada en bienes de equipo que, tras un aumento positivo, regresa a su nivel de estado estacionario transcurridos un reducido número de períodos, salvo, de nuevo en el caso de, en que se amplía sustancialmente el período de pervivencia del sock.

- Por cuanto se refiere al stock de capital privado en equipos (KE) experimenta un claro aumento positivo que perdura de forma sostenida más allá de los cuarenta periodos de referencia que estamos considerando, independientemente de la perturbación que se analice.

- Como consecuencia de lo anterior, la situación del stock de capital privado en infraestructuras (KS), tras las perturbaciones es similar al del stock de capital privado en equipos, es claramente positivo, $\mathrm{y}$ sostenido en el tiempo de referencia.

- Con relación a la fuerza del trabajo (L), la perturbación es más sostenida en el tiempo cuando se refiere a, esto es el gasto público en infraestructuras, lo cual muestra un efecto de corte Keynesiano relevante.

- Los salarios (W) se mantienen en niveles superiores al nivel del estado estacionario cuarenta periodos después independientemente del tipo de stock introducido. No obstante, en el corto plazo se observan algunas diferencias de comportamiento con una leve reducción inicial en el caso del progreso técnico asociado a la inversión privada.

- Tanto la inversión pública en bienes de equipo (IGE), como la inversión pública en infraestructuras (IGS), el stock de capital público en bienes de equipo 
y sistemas de armas (GE) y el stock de capital público en infraestructuras (GS), experimentan un movimiento positivo en el corto plazo, que se mantiene de manera decreciente durante el conjunto de los períodos, de manera que el efecto de las inversiones públicas es uno de los factores que permiten impulsar el crecimiento de forma más clara.
- Tanto el tipo de interés asociado a los bienes de equipo (RE), como el tipo de interés asociado a las infraestructuras (RS), muestran importantes diferencias de comportamiento según sea la perturbación que se considere. No obstante, tienden a situarse por encima del estado estacionario en el largo plazo.

Tabla 5. Análisis comparado de las perturbaciones en las variables.

Resultados del Modelo J

\begin{tabular}{|c|c|c|c|c|c|c|}
\hline \multirow{2}{*}{ Variables } & \multicolumn{2}{|r|}{$A t$} & \multicolumn{2}{|r|}{$Z t$} & \multicolumn{2}{|r|}{$B t$} \\
\hline & $\mathbf{c} / \mathbf{p}$ & $1 / p$ & $\mathbf{c} / \mathbf{p}$ & $1 / p$ & $\mathbf{c} / \mathbf{p}$ & $1 / p$ \\
\hline $\mathbf{Y}$ & + & $\mathrm{EE}(40)$ & + & $\mathrm{EE}(40)$ & + & $\mathrm{EE}(40)$ \\
\hline $\mathrm{C}$ & - & Superior al EE & - & Superior al EE & - & Superior al EE \\
\hline IE & + & $\mathrm{EE}(5)$ & + & $\mathrm{EE}(2)$ & + & $\mathrm{EE}(40)$ \\
\hline IS & + & $\mathrm{EE}(5)$ & + & $\mathrm{EE}(4)$ & + & $\mathrm{EE}(15)$ \\
\hline $\mathbf{K E}$ & + & Superior al EE & + & Superior al EE & + & Superior al EE \\
\hline $\mathbf{K S}$ & + & Superior al EE & + & Superior al EE & + & Superior al EE \\
\hline $\mathbf{L}$ & + & $\mathrm{EE}(3)$ & + & $\mathrm{EE}(3)$ & + & $\mathrm{EE}(10)$ \\
\hline $\mathbf{W}$ & + & Superior al EE & $\begin{array}{l}\text {-inicial/+ } \\
\text { siguiente }\end{array}$ & Superior al EE & + & Superior al EE \\
\hline IGE & + & Superior al EE & + & Superior al EE & + & Superior al EE \\
\hline IGS & + & Superior al EE & + & Superior al EE & + & Superior al EE \\
\hline GE & + & Superior al EE & + & Superior al EE & + & Superior al EE \\
\hline GS & + & Superior al EE & + & Superior al EE & + & Superior al EE \\
\hline $\mathbf{R E}$ & + & $\mathrm{EE}(3)$ & $\begin{array}{l}+ \text { inicial/- } \\
\text { siguiente }\end{array}$ & Inferior al EE & + & Superior al EE \\
\hline RS & - & $\mathrm{EE}(40)$ & - & $\mathrm{EE}(40)$ & - & Superior al EE \\
\hline
\end{tabular}

Notas: +/-: evolución positiva o negativa en el corto plazo. Se considera corto plazo hasta 5 períodos posteriores a la perturbación.

EE(n): Número de períodos transcurridos hasta alcanzar un nuevo Estado Estacionario. Muestra la situación final en el largo plazo.

\section{Conclusiones}

El análisis realizado pretende poner de manifiesto, utilizando un modelo muy simplificador de la realizad, pero plausible, la importancia del gasto público que se destina a estimular la inversión privada como posible generador de efectos positivos sobre el conjunto de la economía. En este sentido, los resultados avalan la hipótesis planteada, observándose cómo aquellas inversiones procedentes del sector públi- co, orientadas a la $\mathrm{I}+\mathrm{D}+\mathrm{i}$, y con una alta incidencia en el progreso tecnológico especifico de la inversión privada, poseen un particular impacto en las empresas privadas que se refleja claramente $y$, con una importante persistencia temporal, con lo que su efecto sobre el PIB se muestra relevante.

En el caso concreto de las firmas que producen bienes para el ámbito de la defensa, debido a su intenso esfuerzo en actividades innovadoras, el efecto del gasto público sería 
especialmente elevado. Así, las políticas orientadas a la adquisición de sistemas de armas cuya complejidad tecnológica es muy elevada y requieren de importantes esfuerzos en I+D, puede generar incrementos de renta elevados y sostenibles temporalmente. Huelga decir que estos incrementos son generadores de empleo y valor añadido.

La financiación de parte de dicho esfuerzo privado por parte del sector público puede suponer un impulso adicional siempre y cuando sea aditivo y no sustitutivo del esfuerzo tecnológico generado por las empresas privadas. Más concretamente, se observa de manera clara que una perturbación de tipo $\mathrm{B}$ asociada con una de tipo $\mathrm{Z}$, muestran que las inversiones públicas en bienes de equipo (como los grandes sistemas de armas) provocan aumentos en la producción y en la mayoría de las variables consideradas.

Así, Los datos calibrados, y mostrados para la elasticidad del capital público en infraestructuras, (Ministerio de Fomento) y los asignados a la elasticidad del capital público en bienes de equipo y sistemas de armas (Ministerio de Defensa) han sido calibrados por primera vez para el caso español, lo cual puede abrir una vía de investigación centrada en los efectos de las inversiones en sistemas de defensa que hasta el momento no han sido realizadas. 


\section{ANEXO}

Tabla A1. Ecuaciones del Modelo J

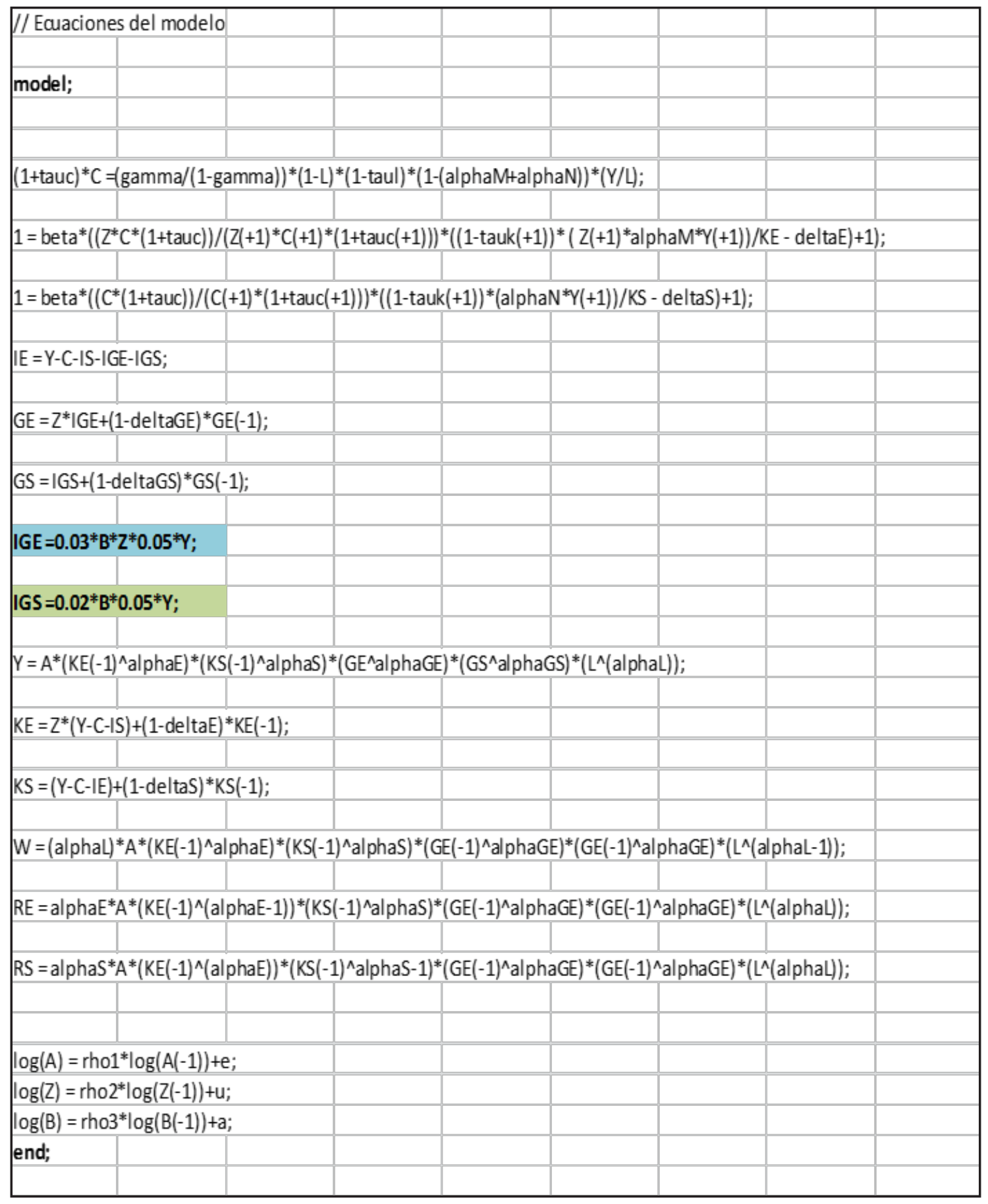


Figura A6. Efectos de las funciones irf tras una perturbación en $Z_{t}$

Figure 1: Orthogonalized shock to e figure 1
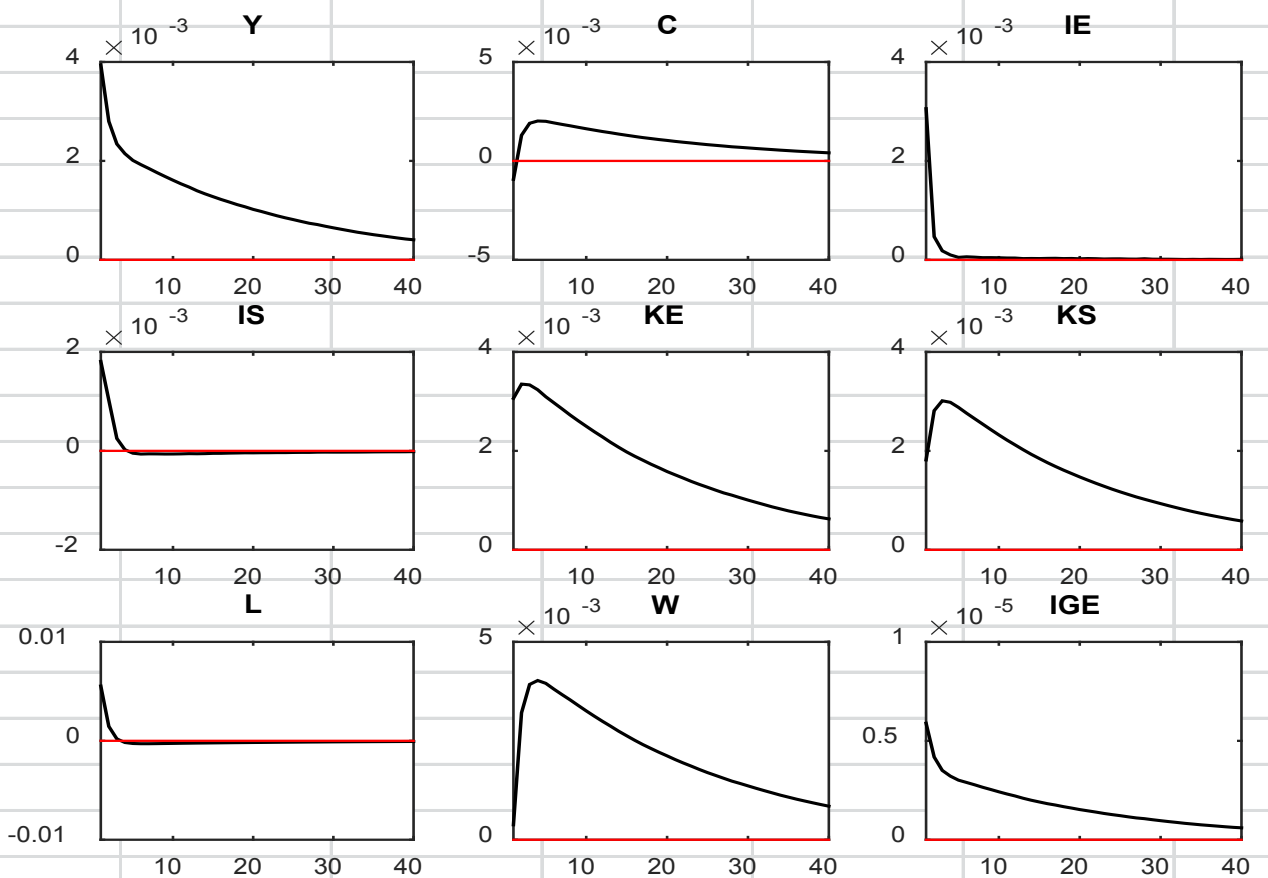

Figure 2: Orthogonalized shock to e figure 2
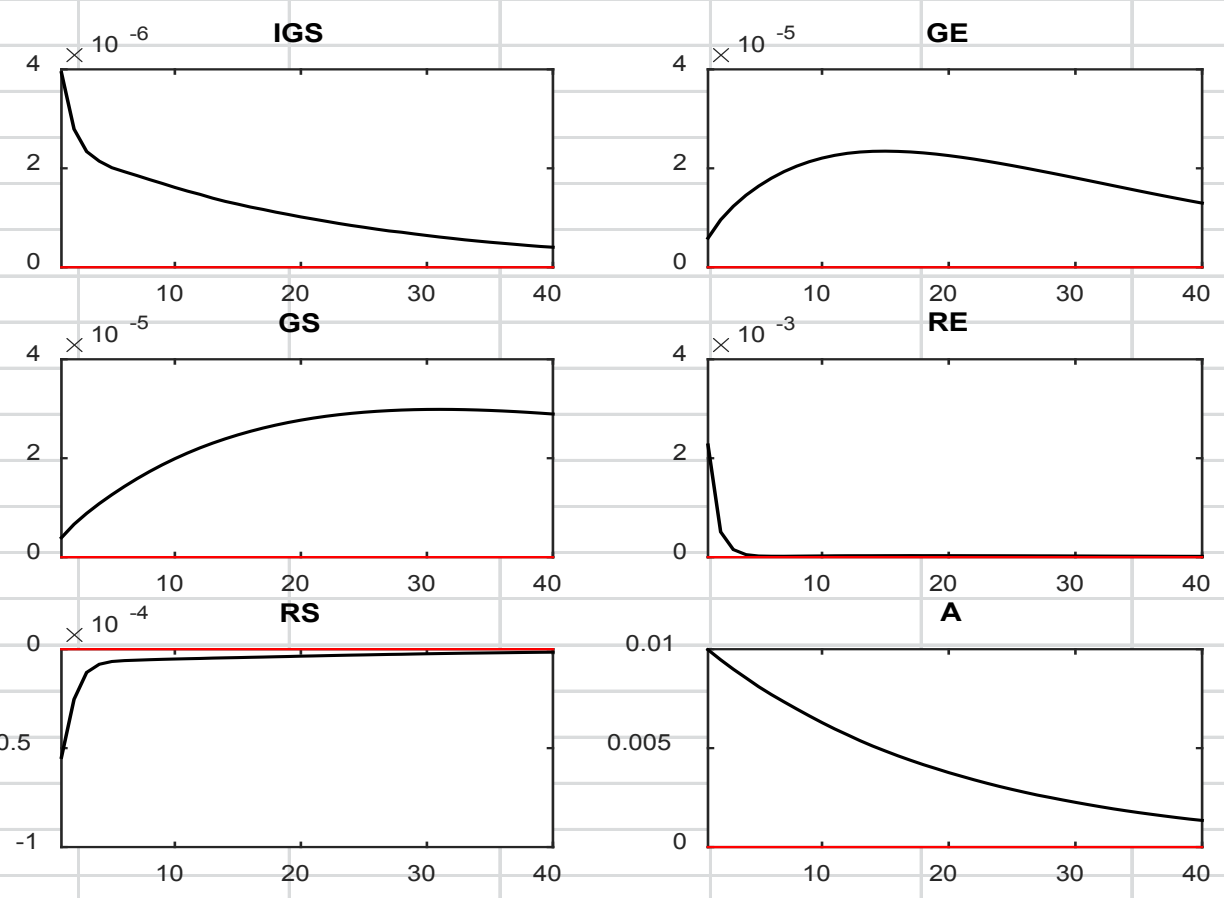
Figura A7. Efectos de las funciones irf tras una perturbación en $Z_{t}$

Figure 3: Orthogonalized shock to u figure 3
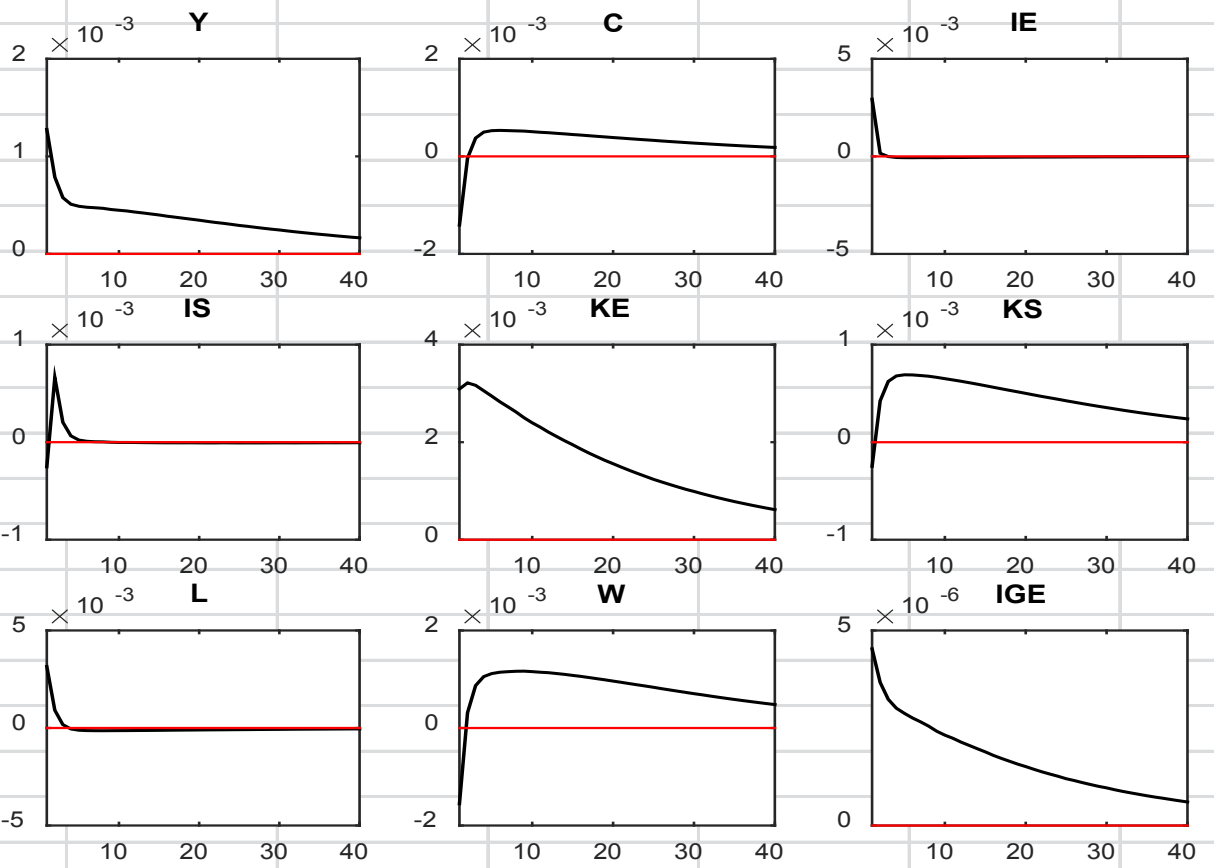

Figure 4: Orthogonalized shock to u figure 4
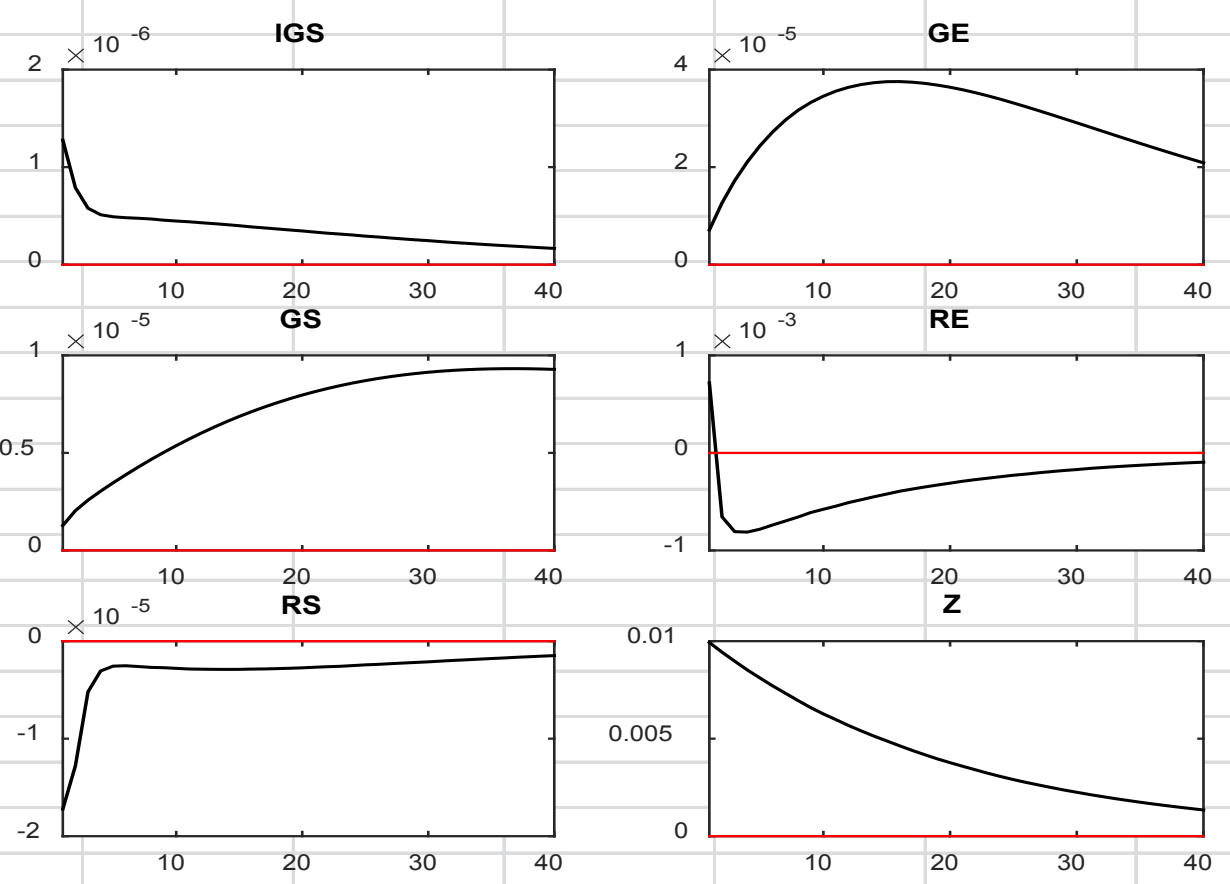
Figura A8. Efectos de las funciones irf tras una perturbación en $B_{t}$

Figure 6: Orthogonalized shock to a figure 6

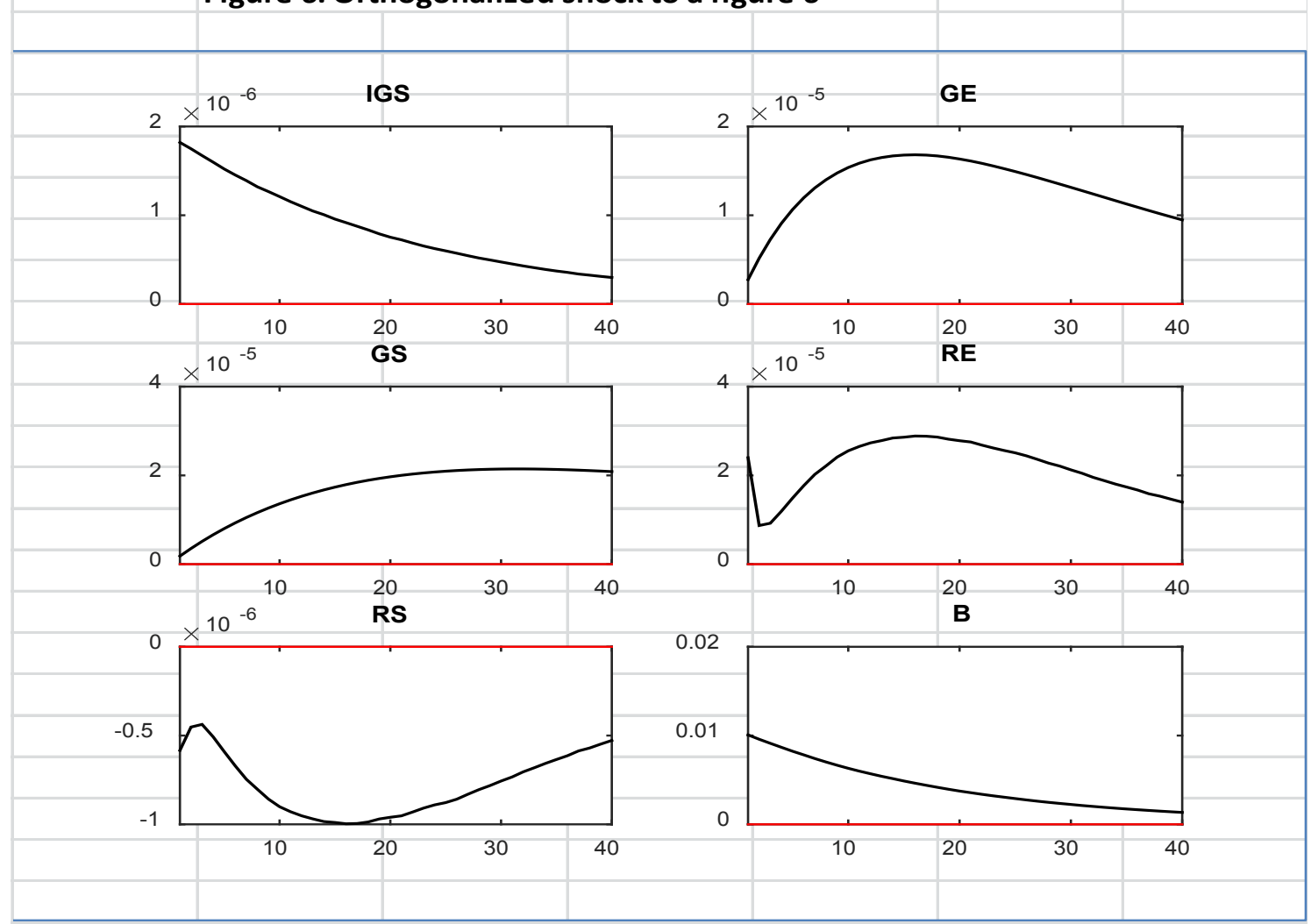

Tabla A2

$\mathrm{Y}=\mathrm{A}^{*}\left(\mathrm{KE}(-1)^{\wedge}\right.$ alphaE $) *\left(\mathrm{KS}(-1)^{\wedge}\right.$ alphaS $) *\left(\mathrm{GE}^{\wedge} \text { alphaGE }\right)^{*}\left(\mathrm{GS}^{\wedge}\right.$ alphaGS $) *\left(\mathrm{~L}^{\wedge}(\right.$ alphaL $\left.)\right) ;$

$\mathrm{W}=(\mathrm{alphaL}) * \mathrm{~A} *\left(\mathrm{KE}(-1)^{\wedge} \mathrm{alphaE}\right) *\left(\mathrm{KS}(-1)^{\wedge} \mathrm{alphaS}\right) *\left(\mathrm{GE}(-1)^{\wedge} \mathrm{alphaGE}\right) *(\mathrm{GE}(-$ $1)^{\wedge}$ alphaGE $)^{*}\left(\mathrm{~L}^{\wedge}(\right.$ alphaL-1 $\left.)\right)$;

$\mathrm{RE} \quad=\quad$ alphaE*A* $\left(\operatorname{KE}(-1)^{\wedge}(\right.$ alphaE-1) $) *\left(\mathrm{KS}(-1)^{\wedge}\right.$ alphaS $) *\left(\mathrm{GE}(-1)^{\wedge} \text { alphaGE }\right)^{*}(\mathrm{GE}(-$ $1)^{\wedge}$ alphaGE $) *\left(\mathrm{~L}^{\wedge}(\right.$ alphaL $\left.)\right)$;

$\mathrm{RS} \quad=\quad$ alphaS*A* $\left(\operatorname{KE}(-1)^{\wedge}(\text { alphaE })\right)^{*}\left(\mathrm{KS}(-1)^{\wedge}\right.$ alphaS-1)*(GE(-1)^alphaGE)*(GE($1)^{\wedge}$ alphaGE $)^{*}\left(\mathrm{~L}^{\wedge}(\right.$ alphaL $\left.)\right)$;

$\log (\mathrm{A})=\operatorname{rho} 1 * \log (\mathrm{A}(-1))+\mathrm{e}$;

Tabla A3

$1=$ beta $^{*}\left((\mathrm{Z} * \mathrm{C} *(1+\operatorname{tauc})) /(\mathrm{Z}(+1) * \mathrm{C}(+1) *(1+\operatorname{tauc}(+1))) *\left((1-\operatorname{tauk}(+1))^{*} \quad \mathrm{Z}(+1) *\right.\right.$ alphaM*Y $\left.(+1)\right) /$

$\mathrm{KE}-$ deltaE)+1);

$\mathrm{GE}=\mathrm{Z} * \mathrm{IGE}+(1-$ deltaGE $) * \mathrm{GE}(-1)$

$\mathrm{KE}=\mathrm{Z} *(\mathrm{Y}-\mathrm{C}-\mathrm{IS})+(1-$ deltaE$) * \mathrm{KE}(-1)$;

$\mathrm{IGE}=0.03 * \mathrm{~B} * \mathrm{Z} * 0.05 * \mathrm{Y}$;

$\mathrm{KE}=\mathrm{Z} *(\mathrm{Y}-\mathrm{C}-\mathrm{IS})+(1-\operatorname{deltaE}) * \mathrm{KE}(-1)$;

Tabla A4

IGE $=0.03 * \mathrm{~B} * \mathrm{Z} * 0.05 * \mathrm{Y}$;

IGS $=0.02 * \mathrm{~B} * 0.05 * \mathrm{Y}$;

$\log (\mathrm{B})=\mathrm{rho} 3 * \log (\mathrm{B}(-1))+\mathrm{a}$; 


\section{Bibliografía:}

C AI, SP CASSOU (1995): “A normative analysis of public capital” Applied Economics 27 (12), 12011209.

ASCHAUER, D. A. (1989): "Is public expenditure productive?", Journal of Monetary Economics, 23: $177-200$.

ARROW, K.J. y KURZ, M. (1970): "Public investment, the rate of return, and optimal fiscal policy", Johns Hopkins Press, Baltimore.

BARRO, R. J. (1990). "Government Spending in a Simple Model of Endogenous Growth". Journal of Political Economy, vol. 98, no. 5, 101-125.

BAXTER, M. Y R.C. KING (1993). " Fiscal Policy in General Equilibrium”. American Economic Review, 83, 315-334.

CASSOU, S. y LANSING, K. (1998), Optimal fiscal policy, public capital and the productivity slowdown, Journal of Economic Dynamics and Control, 22, 911-935.

DE LA FUENTE, A. (2003), El impacto de los Fondos Estructurales: Convergencia real y cohesión interna, Hacienda Pública Española/ Revista de Economía Pública, 165, 129-148.

FINN, M. (1993). Is all government capital productive? Federal Reserve Bank of Richmond Economic Quarterly, 79, 53-80.

GARCÍA ALONSO, J. M. (2010): La base industrial de la defensa en España. Ministerio de Defensa. Madrid.

GLOMM, G. Y B. RAVIKUMAR (1994), "Public investment in infrastructure in a simple growth model", Journal of Economic Dynamics and Control, 18: 1173-1187.

GONZALO F_ de CORDOBA y TORRES, J.L. (2014), "National Security, Military Spending and the Business Cycle" Defence and Peace Economics, 2014.

GREENWOOD, J., HERCOWITZ, Z. y HUFFMAN, G. (1988): Investment, capacity utilization and the real business cycle. American Economic Review 78(3), 402-417.

GREENWOOD, J., HERCOWITZ, Z. y KRUSELL, P. (1997): Long-run -implication of investmentspecific technological change, American Economic Review, 87, 342-362.

GUO, J. y LANSING, K. (1997), Tax structure and welfare in a model of optimal fiscal policy, Economic Review Federal Reserve Bank of Cleveland, 1, 11-23.

HULTEN, C.R. y SCHWAB, R.M. (1993), Infrastructure spending: where do we go from here?, National Tax Journal, 46, 261-273.

JIMENEZ BASTIDA, JOSE LORENZO (2016), Tesis Doctoral "Un análisis macroeconómico de los efectos de la inversión en defensa nacional sobre la base industrial y tecnológica en España”, Universidad Rey Juan Carlos, Facultad de Ciencias Jurídicas y Sociales.

MANZANO, B. (2002): “Inversión pública óptima en un modelo de ciclo real”, Investigaciones económicas, vol. XXXVI (1), 7-34.

MAS, M., MAUDOS, .J., PÉREZ, F. y URIEL, E. (1996), Infrastructures and productivity in the Spanish regions, Regional Studies, 30, 641-649.

PAVITT, K. y PATEL, P. (1988) "The International Distribution and Determinants of Technological Activities". Oxford Review of Economic Policy, vol.4, n ${ }^{\circ}$ 4, 35-55.

TORRES, J. L. (2009): Capital público y crecimiento económico en España 1980-2004. Hacienda Pública Española/Revista de Economía Pública, 188(1), 31-53.

TORRES, J. L., (2013): "Introduction to Dynamic Macroeconomic General Equilibrium Models". Vernon Series in Economic Methodology. 